\title{
Single-cell atlas reveals meningeal leukocyte heterogeneity in the developing mouse brain
}

\author{
Aura Zelco, ${ }^{1}$ Vanja Börjesson, ${ }^{2}$ Jurrian K. de Kanter, ${ }^{3}$ Cristina Lebrero-Fernandez, ${ }^{4}$ \\ Volker M. Lauschke, ${ }^{5,6}$ Eridan Rocha-Ferreira, ${ }^{7}$ Gisela Nilsson, ${ }^{1}$ Syam Nair, ${ }^{7}$ Pernilla Svedin, ${ }^{1}$ \\ Mats Bemark, ${ }^{4}$ Henrik Hagberg, ${ }^{7}$ Carina Mallard, ${ }^{1}$ Frank C.P. Holstege, ${ }^{3}$ and Xiaoyang Wang ${ }^{1,7,8}$ \\ ${ }^{1}$ Centre of Perinatal Medicine and Health, Institute of Neuroscience and Physiology, Department of Physiology, Sahlgrenska \\ Academy, University of Gothenburg, Gothenburg 40530, Sweden, ${ }^{2}$ Bioinformatics Core Facility, Sahlgrenska Academy, University \\ of Gothenburg, Gothenburg 413 90, Sweden; ${ }^{3}$ Princess Máxima Center for Pediatric Oncology, 3584 CS Utrecht, The Netherlands; \\ ${ }^{4}$ Department of Microbiology and Immunology, Sahlgrenska Academy, University of Gothenburg, Gothenburg 40530, Sweden; \\ ${ }^{5}$ Department of Physiology and Pharmacology, Karolinska Institute, Stockholm 17177, Sweden; ${ }^{6}$ Dr. Margarete Fischer-Bosch \\ Institute of Clinical Pharmacology, Stuttgart 70376 , Germany; ${ }^{7}$ Centre of Perinatal Medicine and Health, Institute of Clinical \\ Sciences, Department of Obstetrics and Gynecology, Sahlgrenska Academy, Gothenburg University, Gothenburg 40530, Sweden; \\ ${ }^{8}$ Henan Key Laboratory of Child Brain Injury, Institute of Neuroscience, Third Affiliated Hospital of Zhengzhou University, \\ Zhengzhou 450052, China
}

The meninges are important for brain development and pathology. Using single-cell RNA sequencing, we have generated the first comprehensive transcriptional atlas of neonatal mouse meningeal leukocytes under normal conditions and after perinatal brain injury. We identified almost all known leukocyte subtypes and found differences between neonatal and adult border-associated macrophages, thus highlighting that neonatal border-associated macrophages are functionally immature with regards to immune responses compared with their adult counterparts. We also identified novel meningeal microglia-like cell populations that may participate in white matter development. Early after the hypoxic-ischemic insult, neutrophil numbers increased and they exhibited increased granulopoiesis, suggesting that the meninges are an important site of immune cell expansion with implications for the initiation of inflammatory cascades after neonatal brain injury. Our study provides a single-cell resolution view of the importance of meningeal leukocytes at the early stage of development in health and disease.

[Keywords: preterm brain injury; neonatal mouse; meningeal leukocytes; single-cell RNA sequencing]

Supplemental material is available for this article.

Received December 17, 2020; revised version accepted June 28, 2021.

The meninges are part of the central nervous system (CNS) and consist of a triple-layer barrier divided into the dura, arachnoid, and pia mater. These membranes protect from potential threats coming from the periphery that might affect the brain parenchyma. Recent studies have revealed the role of meningeal resident and infiltrating immune cells in response to different stimuli under physiological conditions and in brain pathologies (Mrdjen et al. 2018; Van Hove et al. 2019; Utz et al. 2020). However, little is known about the role of neonatal meningeal immune cells in CNS development and pathology, though

Corresponding authors: xiaoyang.wang@fysiologi.gu.se, aura.zelco@gmail.com

Article published online ahead of print. Article and publication date are online at http://www.genesdev.org/cgi/doi/10.1101/gad.348190.120. Article freely available online through the Genes \& Development Open Access option. the development of meningeal fibroblast diversity and function have been studied (DeSisto et al. 2020).

The first postnatal week in rodents is characterized by rapid development of the CNS, similar to development in humans during late pregnancy and the perinatal period. Postnatal days (PND) 2-7 in mice correspond to gestational weeks 23-32 in humans (Mallard and Vexler 2015), a stage with the greatest vulnerability to white matter damage, which is one of the most common types of injury in preterm infants (Back 2017; Volpe 2019). This is because premyelinating oligodendrocytes at this developmental stage are most abundant, and they are highly susceptible

(C) 2021 Zelco et al. This article, published in Genes \& Development, is available under a Creative Commons License (Attribution-NonCommercial 4.0 International), as described at http://creativecommons.org/licenses/by-nc/4.0/. 
to insults (Back et al. 2001). Preterm brain injuries, such as encephalopathy of prematurity, can lead to cognitive and motor disabilities. There is still no effective treatment for these conditions, although therapeutic approaches such as erythropoietin have been tested (Song et al. 2016; Juul et al. 2020). The pathogenesis of preterm brain injury is complex, and we and others have shown that immune cells play key roles in the development of brain injury (Zhang et al. 2017; Albertsson et al. 2018; Herz et al. 2018; Nazmi et al. 2018). After insult in the neonatal brain, $\gamma \delta$ T cells have a detrimental effect by enhancing brain damage, more so than conventional $\mathrm{T}$ and $\mathrm{B}$ cells (Zhang et al. 2017; Albertsson et al. 2018; Nazmi et al. 2018), which differs from the adult brain where both $\gamma \delta \mathrm{T}$ cells (Shichita et al. 2009) and conventional T cells (Yilmaz et al. 2006; Hurn et al. 2007) play important roles in ischemic injury. The $\gamma \delta \mathrm{T}$ cells tend to concentrate in the meninges after brain injury (Albertsson et al. 2018; Alves de Lima et al. 2020) similar to innate lymphoid cells (ILCs) (Gadani et al. 2017; Zelco et al. 2020). However, we still know little about the immune landscape of the meninges in newborns, including whether or not the meninges represent an important site harbouring immune cells that take part in the inflammatory response and contribute to injury processes after insult to the developing brain.

The purpose of this study was to reveal the heterogeneity and transcriptomic signature of resident leukocyte populations in the neonatal mouse meninges using droplet-based single-cell RNA sequencing (scRNA-seq). We also investigated the acute effect of hypoxia-ischemia (HI)-induced preterm brain injury on the meningeal leukocyte composition and the dynamics of single cell expression signatures.

\section{Results}

The neonatal mouse meningeal leukocyte population is heterogeneous

To study resident and infiltrating leukocytes in the neonatal meninges, we sorted meningeal leukocytes expressing intermediate and high levels of CD45 (CD45 $5^{\text {int+high) from }}$ PND4 naïve and HI pups $6 \mathrm{~h}$ after the insult and performed scRNA-seq (Fig. 1A; Supplemental Fig. S1). To identify the cell populations, we used both a manual method with CellMarker in Seurat and an automatic annotation using CHETAH (characterization of cell types aided by hierarchical clustering) (Fig. 1B,C; Supplemental Data 1; de Kanter et al. 2019). Stratification by markers for immune and neural cell populations identified seven clusters: border-associated macrophages (BAMs), microglia-like cells, monocytes, neutrophils, T cells, ILCs, and B cells (Fig. 1B,C). We crossexamined the two annotation methods and confirmed the consistency between annotations (Fig. 1D).

BAMs appeared as the main cluster $(15,583$ cells $)$, followed by microglia-like cells (2822 cells) and monocytes (2012 cells), while neutrophils, T cells, B cells, and ILC clusters were much smaller $(401,201,304$, and 240 cells, respectively) (Fig. 1E; Supplemental Tables S1, S2). Each cluster expressed highly specific marker genes for the cor- responding cell types, which agreed with the manual annotation (Fig. 1F,G; Supplemental Data 1). All cell clusters expressed varying levels of Ptprc, the gene encoding CD45 (Fig. 1G), confirming that the leukocyte preparations were not contaminated with other neural cells.

BAMs, microglia-like cells, and monocytes are distinct yet intertwined cell populations

A recent study characterized BAMs during embryonic development (Utz et al. 2020), where BAMs were defined by the expression of Mrc1 (CD206), Lyve1, Ms4a7, and Pf4. Similar to BAMs from embryonic mouse meninges, the neonatal mouse meningeal BAM cluster expressed the typical signature markers Mrc1, Lyve1, Ms4a7, and Pf4 (Fig. 2A-D). All four markers were mainly expressed in the BAM cluster, with some microglia-like cells positive for Mrc1 or Lyve1 (Fig. 2A,B, Supplemental Fig. S2A,B). Therefore, BAMs in the neonatal meninges shared the signature genes common to BAM populations in the embryonic mouse. By immunofluorescence staining of markers for BAMs (CD206 and Lyve1) and microglia (Iba-1), we indeed confirmed that the majority of macrophages were BAMs $\left(\mathrm{CD} 206^{+} / \mathrm{Iba}^{+}{ }^{+} / \mathrm{Lyve}^{+}\right)$in the neonatal meninges, while microglia-like cells $\left(\mathrm{CD} 206^{-} / \mathrm{Iba}^{+}{ }^{+} / \mathrm{Lyve}^{+}\right)$were also present (Fig. 2E).

The BAM cluster comprised several subclusters (Fig. 2F). To explore this further, we compared the neonatal meningeal BAMs with the existing BAM database generated from adult mouse meninges (Van Hove et al. 2019), in which the meningeal BAM population was subdivided based on the expression level of signature genes. We also included the monocyte clusters to determine whether this was a population distinct from BAMs as previously described (Van Hove et al. 2019). We aggregated the data from the neonatal meningeal BAMs and monocyte clusters with the adult mouse meningeal BAM and monocyte data set (Van Hove et al. 2019) obtained from enriched subdural (SD) and dural (D) meninges, which are the anatomical structures most similar to the meninges used in the current study. Neonatal meningeal BAMs mainly overlapped with SD-BAMs from the adults, while overall very few D-BAMs were present (Fig. 2G). However, SDBAMs, D-BAMs, and their subtypes D2 or D3, as found in the adult meningeal BAMs, did not segregate when analyzing the neonatal BAM population alone (Fig. 2H-J; Supplemental Fig. S2D-G). These results are in accordance with previous findings (Mrdjen et al. 2018; Van Hove et al. 2019), indicating that BAM heterogeneity is very low at birth and increases with age and that neonatal BAMs mainly express low levels of MHCII (MHCII low) (Supplemental Fig. S2F). The neonatal and adult monocyte populations overlapped quite well, as expected, although they still showed some differences, especially in subcluster 2 (Fig. 2G; Supplemental Fig. S3A). Additionally, we observed that the neonatal meningeal monocyte cluster expressed signature genes typical for adult D2 and D3 BAMs (Fig. 2I,J). These results, together with the vicinity of adult monocyte subcluster 2 and D-BAMs, may indicate that at least part of the adult D-BAMs might 


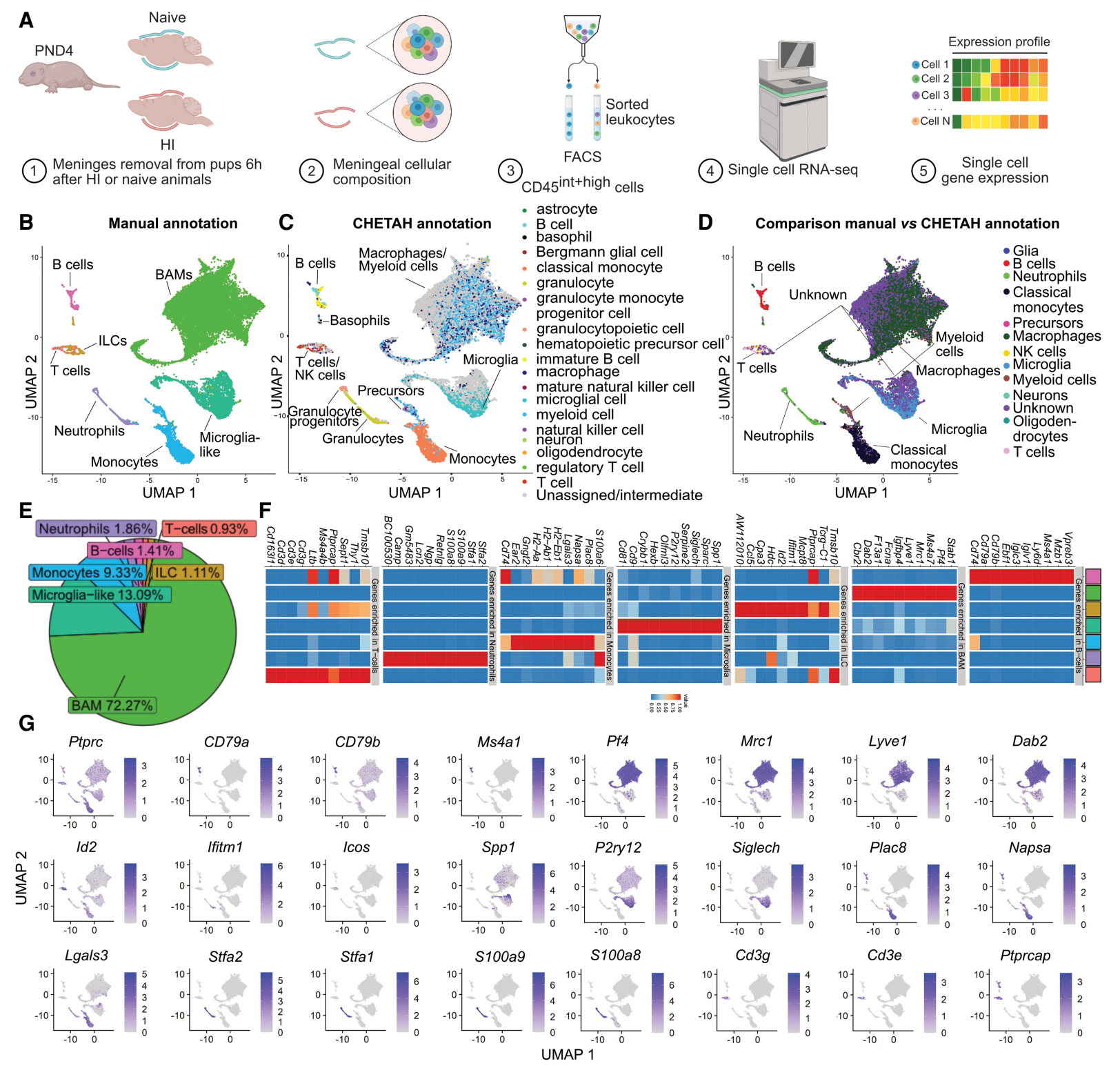

Figure 1. The meningeal leukocyte population is composed of several subtypes of immune cells with typical signature marker genes. (A) Dissection of the meninges from PND4 naïve mice and at $6 \mathrm{~h}$ after HI, and the schematic overview of the 10X Chromium scRNA-seq procedure (created with Biorender.com). $(B, C)$ Uniform manifold approximation and projection (UMAP) plot generated using Seurat $(B)$ or CHETAH $(C)$. Confidence score $>0.1$. (D) Overlay of CHETAH annotations in the Seurat UMAP. $(E)$ Numbers expressed as percentages of each cell population among the total neonatal meningeal leukocytes. $(F)$ Heat map showing the signature gene expression for each cluster identified in $B .(G)$ UMAP showing the expression of some signature genes present in the heat map.

have a monocyte origin instead of maturing from macrophages, as suggested previously (Van Hove et al. 2019; Utz et al. 2020). Trajectory analysis including the neonatal meningeal BAMs and monocyte clusters (subclusters 1 and 2) as well as the corresponding adult dural BAMs and monocyte clusters confirmed that, overall, the neonatal clusters tracked well with their adult counterparts, both for BAMs and monocytes (Fig. 2K). Interestingly, neonatal and adult monocyte subclusters 2 were shown to be intermediate populations between monocyte subcluster 1 and BAMs. This indicates that monocyte sub- cluster 2 might be one of the sources that gives the mixed ontogeny of the adult dural BAMs as previously observed (Van Hove et al. 2019).

BAMs, monocytes, and microglia-like cells share several common markers and are usually difficult to distinguish unambiguously, so we conducted a trajectory analysis to identify whether and in what way neonatal meningeal BAMs, microglia-like cells, and monocyte cell clusters were related to each other. To test whether we could discern the different subclusters as well as microglia-like cells and monocytes, we included all 


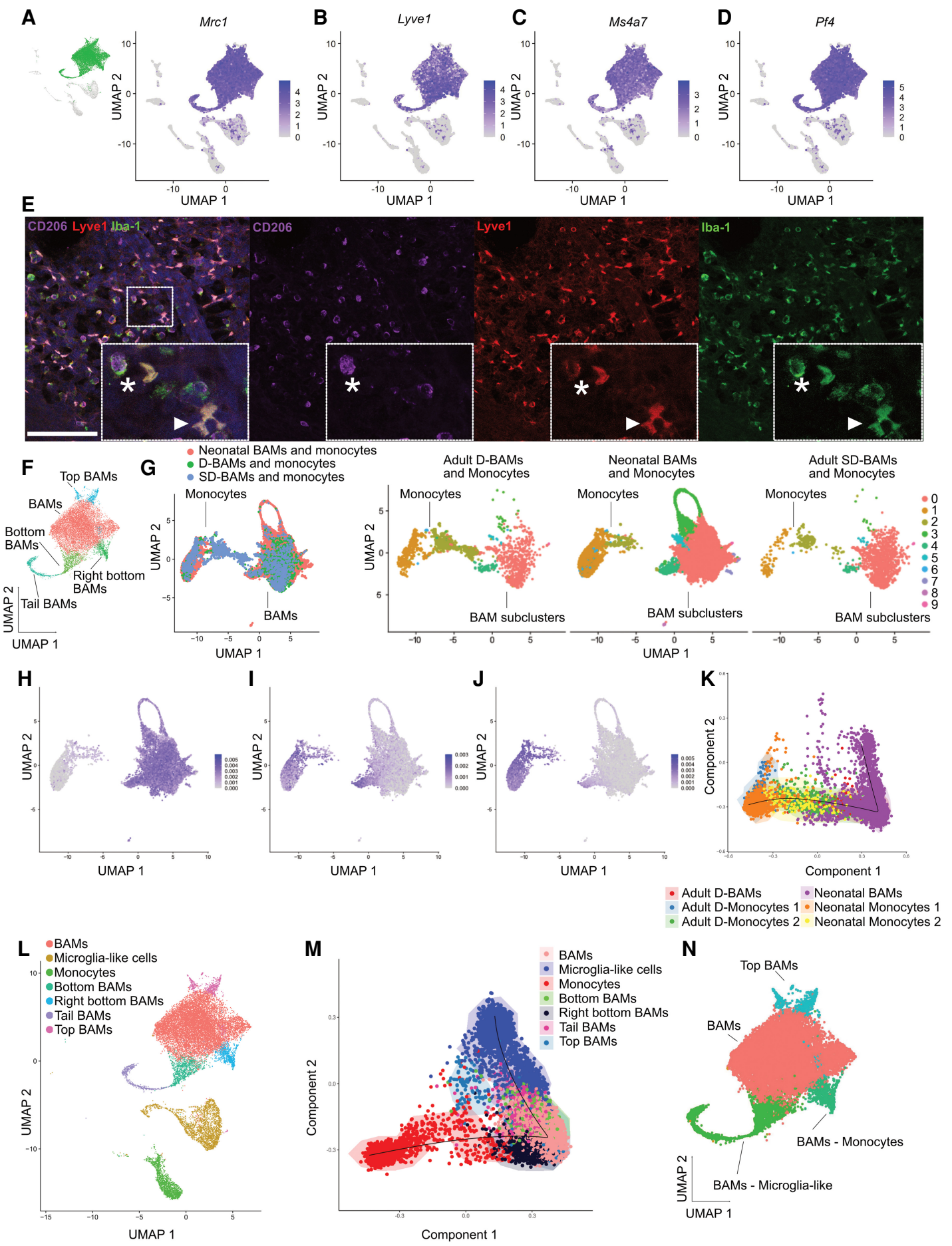

Figure 2. Gene expression and characterization of neonatal BAMs compared with embryonic and adult BAMs, monocytes, and microglia-like cells. $(A-D)$ Gene expression of $\operatorname{Mrc1}(A)$, Lyve1 $(B), M s 4 a 7(C)$, and $P f 4(D)$, which are typical BAM markers. (E) Immunofluorescent staining of BAMs (CD206 $/$ Lyve $1^{+} / \mathrm{Iba}^{-1^{+}}$, star) and microglia-like cells (CD206 ${ }^{-} \mathrm{Lyve}^{+} / \mathrm{Iba}-1^{+}$, arrowhead) in naïve meninges at PND4. Scale bar, $100 \mu \mathrm{m}$. (F) Subclustering of BAMs in Seurat. (G) Comparison of neonatal BAMs and monocytes with BAMs and monocytes from adult SD and D meninges. The left UMAP shows the neonatal BAMs and monocytes with SD-BAMs and D-BAMs overlaid, while the right plots are the split view of the same UMAP based on the source of the cells. $(H-J)$ Signature gene expressions that are typical of adult SD-BAMs (Ccr1, Lyve1, Ednrb, Colec12, Prps2, Ptgds, P2rx7, and Egf17; H), adult D-BAMs subtype 2 (Irf7, Crip1, and Cc19; I), and adult D-BAMs subtype 3 (Clec4b1, Ccr2, Vim, Lsp1, and Lgals3; $J$ ) in the neonatal data set. (K) SCORPIUS trajectory analysis to study the relationships among neonatal BAMs and monocytes (divided as subclusters 1 and 2) and the corresponding adult subclusters from the dural data set. (L) UMAP plot showing microglia-like cells, monocytes, and subclusters of BAMs. (M) SCORPIUS trajectory analysis showing the relationship between BAM subclusters, microglia-like cells, and monocytes. $(N)$ New BAM subclustering after trajectory analysis. 
apparent subclusters of BAMs (Fig. 2L). SCORPIUS analysis showed that neonatal meningeal monocytes, microglia-like cells, and BAMs were three distinct populations and that BAMs were in the middle of the other two populations in terms of gene expression (Fig. 2M), although a few cells showing intermediate gene expression between BAMs and microglia-like cells were also observed. To note, we did not observe obvious differences between the subclusters of BAMs (Fig. 2M,N). Therefore, there is a population of BAMs in the neonatal mouse meninges that has a similar gene signature compared with adult SD-BAMs. Additionally, the neonatal meningeal BAM population was distinct from monocytes and microglialike cells, although some cells may be an intermediate subpopulation between BAMs and microglia-like cells, and to a lesser extent between BAMs and monocytes, having gene expression properties of both cell types

Neonatal BAMs show cytoskeleton and immune response-related developmental changes compared with adult $B A M s$

Once we established the presence of a BAM cluster comprising a distinctly separate cell population, we characterized those cells in more depth to highlight potential developmental differences between neonatal BAMs and adult BAMs from the literature (Fig. 3A; Van Hove et al. 2019). There were $>600$ differentially expressed genes between neonatal naive BAMs and adult BAMs (SD-BAMs and D-BAMs) (Supplemental Data 2). The top 20 up-regulated genes were involved in ATP production in mitochondria (mt-Atp8 and Atp5g3) and cell growth (Lyve1 and Igfbp4) (Supplemental Table S3), while the most down-regulated genes were related to attraction to monocytes and basophils (Ccl2), antigen binding (H2-Ab1 and $H 2-A a)$, and antigen-presenting cell response $(C d 74)$ (Supplemental Table S4). Significant gene ontology (GO)-enriched biological processes included metabolism, actin cytoskeleton organization, and oxidoreductase activity (Fig. 3C; Supplemental Data 3). These findings were further confirmed by ingenuity pathway analysis (IPA), with cytoskeleton-related signaling, the Eif 2 signaling pathway, and oxidoreductase activity among the top 20 up-regulated canonical pathways, while signaling pathways related to inflammation and immune responses were dominant within the top 20 down-regulated canonical pathways (Fig. 3D). In addition, Setd2 was predicted to be the regulator of microtubule dynamics and necrosis through down-regulation of the Jun, Rhob, and Rtn4 genes (Fig. 3E). The down-regulation of Jun and $R h o b$, along with other genes, was also predicted to result in down-regulation of immune response processes (for example, the binding of myeloid cells), with the proto-oncogene $M y c$ being among the main upstream regulators affecting Jun and Rhob expression (Fig. $3 F-G)$. Overall, BAMs in neonatal mice showed up-regulation of cytoskeleton-related signaling and cellular respiration compared with adult mice, while other functional processes that are characteristic of mature macrophages, such as the recruitment of cells and immune response activity, were down-regulated compared with adult mice.
Neonatal meningeal monocytes show different immune responses compared with their adult counterparts

Next, we investigated differences between adult and neonatal meningeal monocytes (Fig. 2G), specifically studying the developmental changes between these two populations. We found a different distribution of cells in neonatal versus adult monocytes (Supplemental Fig. S3A). There were 584 differentially expressed genes between neonatal and adult meningeal monocytes (Supplemental Fig. S3B; Supplemental Tables S5, S6), and the GO term enrichment analysis showed an up-regulation of immune-related processes in neonatal monocytes compared with adult monocytes (Supplemental Fig. S3C; Supplemental Data 3). IPA confirmed these results, showing an up-regulation of immune signaling and cytoskeleton pathways, such as leukocyte extravasation and integrin signaling (Supplemental Fig. S3D). Eif2 signaling was among the down-regulated pathways, and when this pathway is defective cells become more susceptible to bacterial invasion (Shrestha et al. 2012). Thus, these analyses suggest that neonatal meningeal monocytes, although having increased immune-related processes compared with adults, may still be more susceptible to bacterial invasion.

The neonatal meningeal microglia-like cell population shows different regulation of the immune response and distinct features compared with parenchymal microglia

As previously established, microglia-like cells formed a distinct cluster from BAMs. Sall1, a microglia-specific gene, was observed in the microglia-like cell populations but not in BAMs (Fig. 4A; Supplemental Fig. S2H). Also, Tgfbr1, which is necessary for microglial development (Utz et al. 2020), was expressed predominantly in microglia-like cells (Fig. 4B). Microglia-like cells also expressed Aif-1 (Iba-1), Itgam (Cd11b), and Itgb2 (Cd18) (Supplemental Fig. S2I-K). Iba-1 is a typical microglial marker, while the integrins $\mathrm{Cd} 11 \mathrm{~b}$ and $\mathrm{Cd} 18$ form complement receptor 3 , which is expressed exclusively by microglia (Akiyama and McGeer 1990; Stephan et al. 2012) and is involved in synaptic phagocytosis by microglia (Stevens et al. 2007; Schafer et al. 2012). Therefore, the meningeal microgliallike cells observed may retain typical functions of parenchymal microglia.

The microglia-like cell population could be reclustered into several subpopulations (Fig. 4C). To identify features of subpopulations and to confirm that these cells were not contaminants from the parenchymal microglia, we aggregated and compared neonatal meningeal microglia-like cells with parenchymal microglia from PND4/5 mice (Hammond et al. 2019), the same age as the mice used in the current study. The two data sets overlapped to a large extent, and all 14 subclusters from the aggregated data could be found in both data sets separately (Fig. 4C,D). Hammond et al. (2019) identified a total of five subpopulations of microglia highly specific for this age, characterized by the expression of several markers. Four out of the five main parenchyma microglia subclusters in the PND4/5 mouse brain were found in the aggregated data set, as 


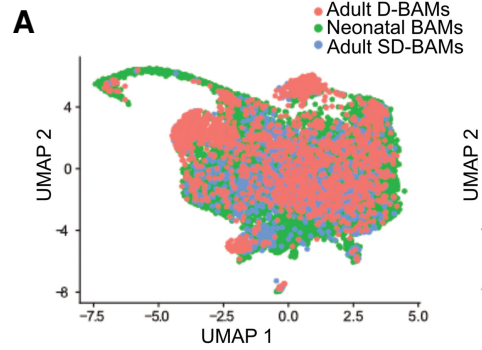

B

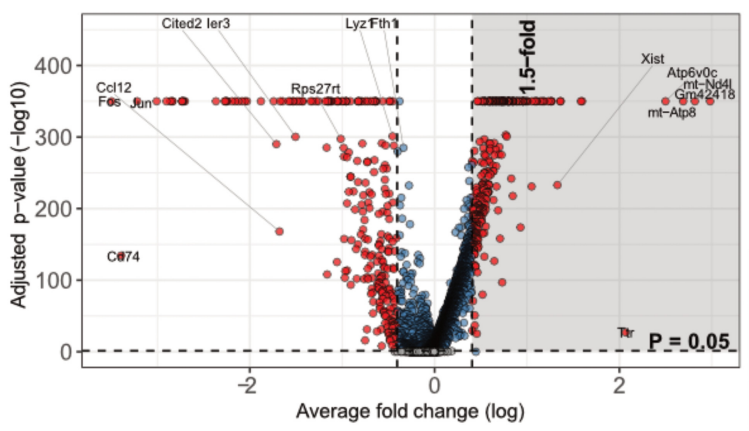

cat $•$ Intermed $\odot$ Non-sign • Sign
Adult D-BAMs Neonatal Naive BAMs

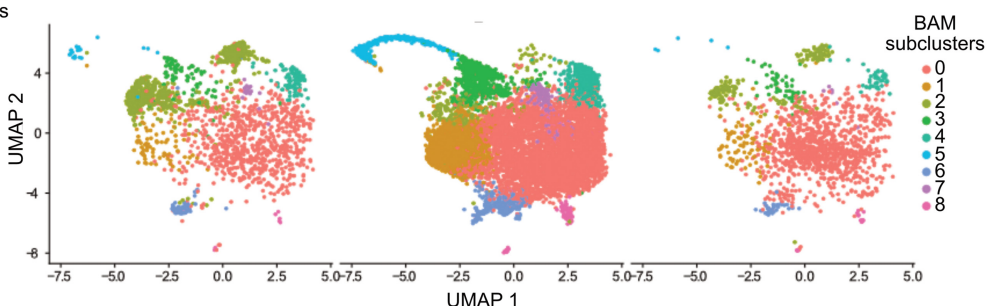

C
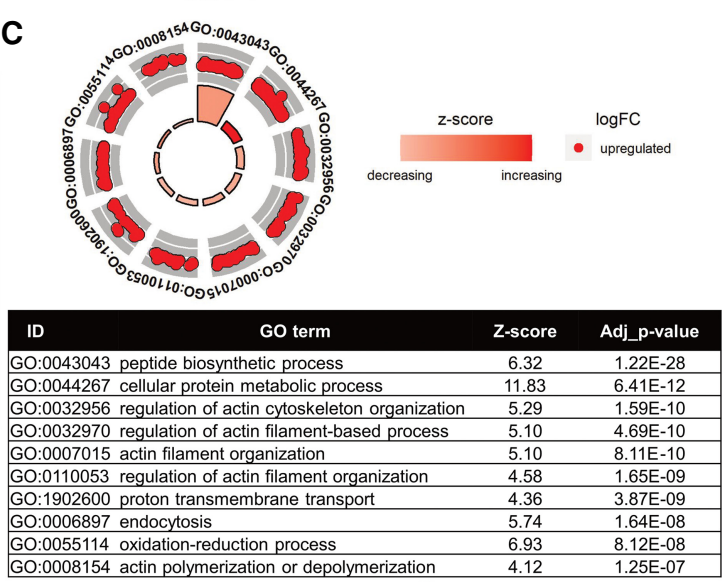

D z-scores

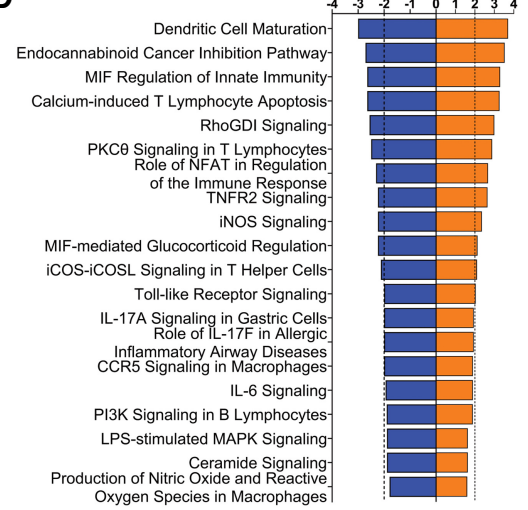

Actin Cytoskeleton Signaling Integrin Signaling Regulation of Actin-based Motility by Rho EIF2 Signaling in Macrophages and Monocytes
Oxidative Phosphorylation RhoA Signaling HOTAIR Regulatory Pathway Ephrin Receptor Signaling Rac Signaling Actin Nucleation by ARP-WASP Complex Cdc42 Signaling PI3K/AKT Signaling Paxillin Signaling Reelin Signaling in Neurons eNOS Signaling Ga12/13 Signaling Apelin Adipocyte Signaling Pathway Synaptogenesis Signaling Pathway

E

$\mathbf{F}$

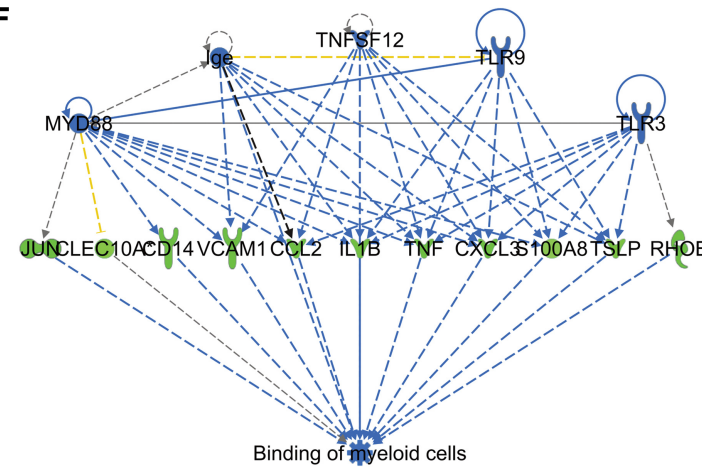

G

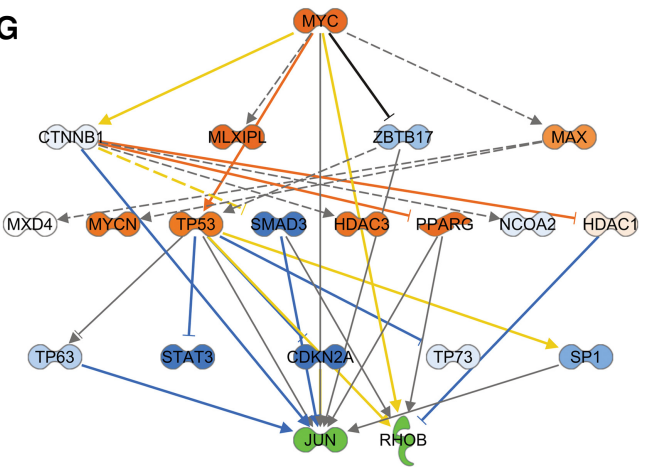

Figure 3. Neonatal BAMs have increased gene expression for cytoskeleton processes, while immune response is down-regulated compared with adults. (A) UMAP of neonatal naive BAMs and the overlap with adult SD-BAMs and D-BAMs. The left UMAP is the overlay from the SD-BAMs and D-BAMs, while the right UMAP shows the split of SD-BAMs and D-BAMs in the neonatal BAM cluster. (B) Volcano plot for differentially expressed genes between neonatal and adult BAMs, with genes highlighted in the gray area having fold change $>1.5$ and $P<0.05$ in the neonatal BAMs compared with adult BAMs. (C) GOPlot representation of relevant GO terms from the gProfiler analysis, with the table abbreviations below. The outer circle represents how many genes are up-regulated or down-regulated in the GO term. The inner circle has a double function: The height of the bar indicates the negative $\log _{10}$ adjusted $P$-value (the taller, the more significant), and the color of the bar represents the $z$-score. $(D-G)$ IPA canonical pathways analysis. $(D) z$-score $>1.5, P<0.05$ for all. Regulator analyses for Setd2 $(E)$ and other genes involved in binding of myeloid cells $(F)$. (G) Upstream regulator analysis showing the effects of $M y c$ activation. 

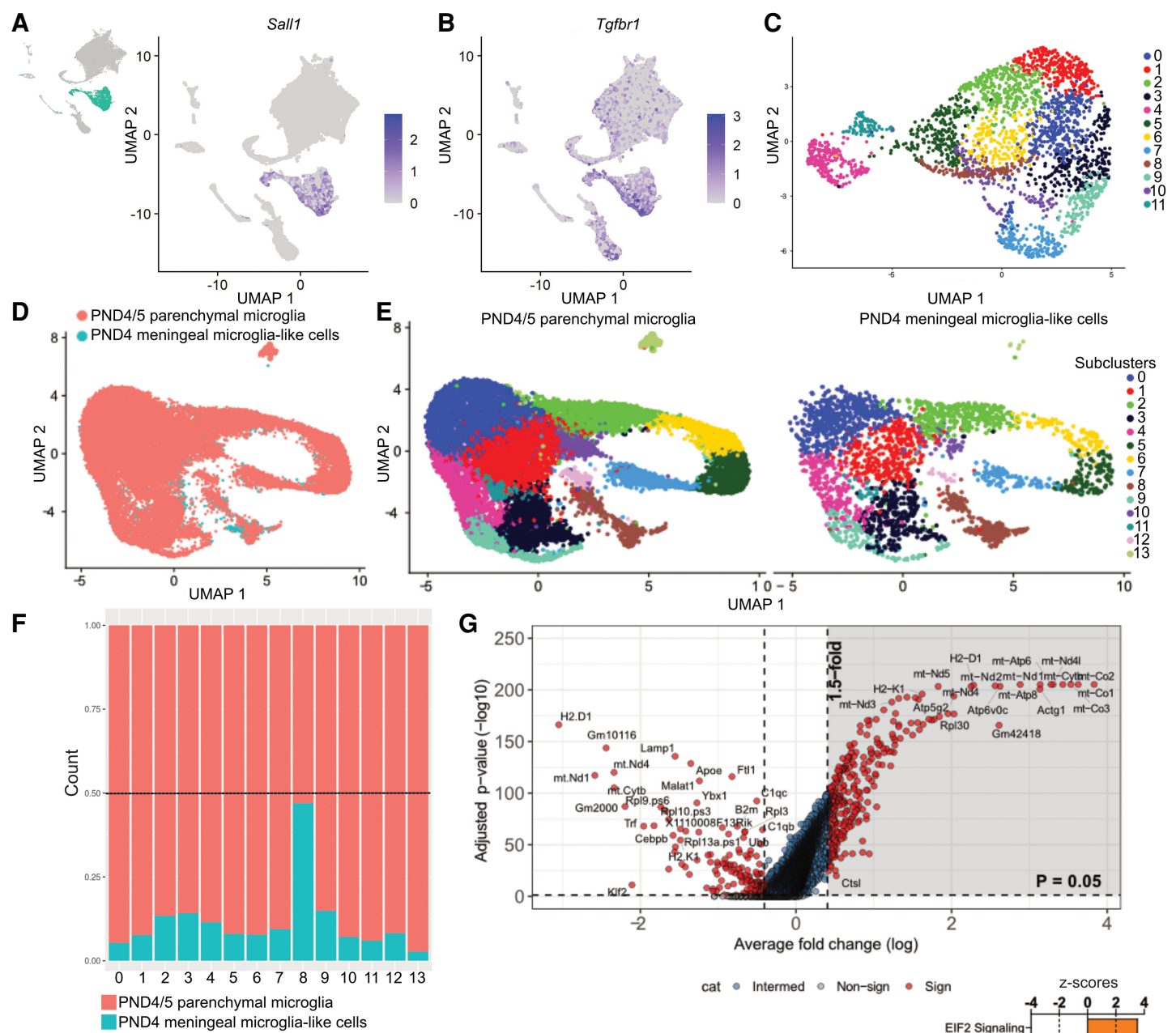

H
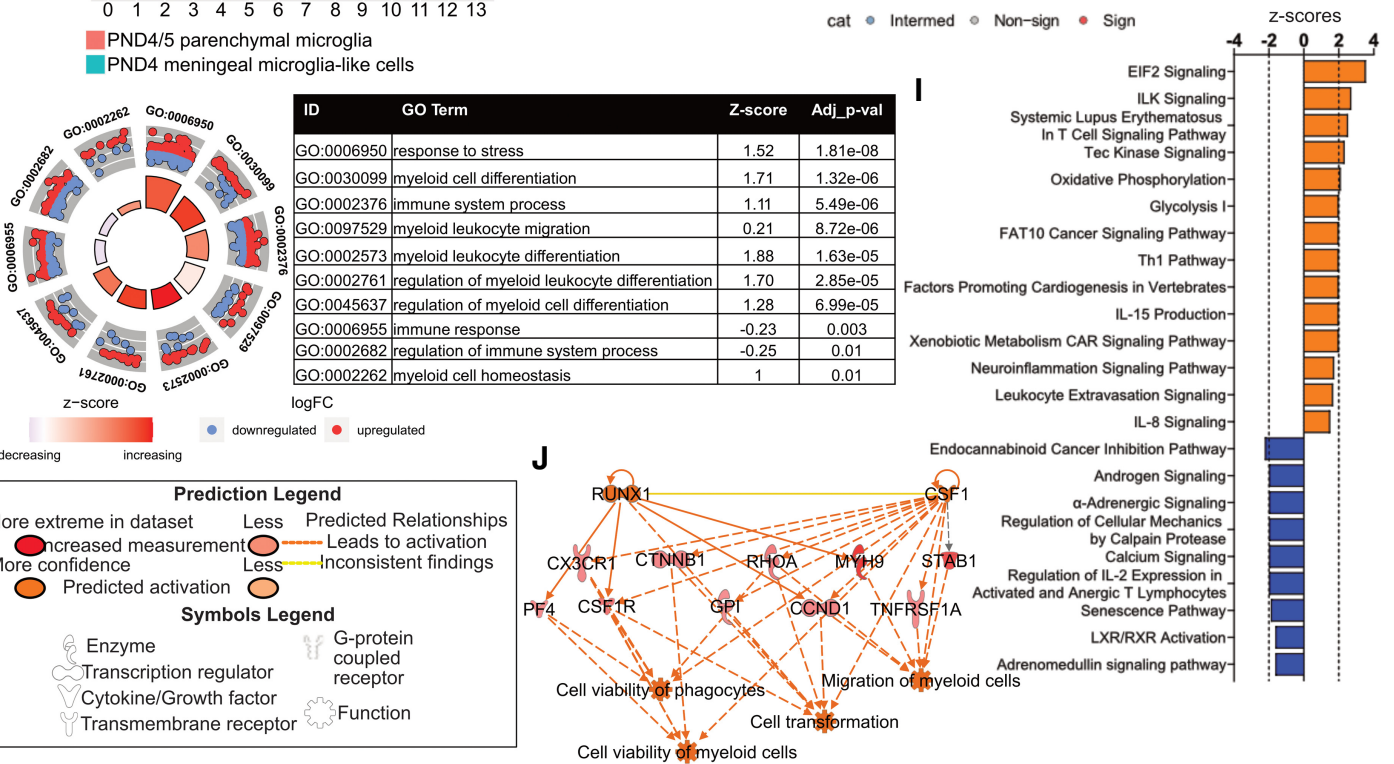

Figure 4. Meningeal microglia-like cells show different frequencies of subpopulations compared with parenchymal microglia. $(A, B)$ UMAP plots showing gene expression of Sall1 $(A)$ and $\operatorname{Tg} f b r 1(B)$. $(C)$ UMAP plots showing the initial meningeal microglia-like cell subpopulations. $(D, E)$ UMAP plots showing the aggregation analysis results of the meningeal microglia-like cells with parenchymal microglia from PND4/5 mice (Hammond et al. 2019) as the overlap of two data sets shown in $D$ and the 14 subclusters found in both data sets (parenchymal microglia on the left and meningeal microglia-like cells on the right) shown in $E$. ( F) Frequency bar chart showing the proportion of the microglia subclusters in the meningeal and parenchymal data set. $(G, H)$ Differentially expressed gene analysis for aggregation subcluster 8 comparing meningeal and parenchyma cells, presented as a volcano plot $(G)$ and GOPlot representation of relevant GO terms from the gProfiler analysis $(H)$. The outer circle represents how many genes are up-regulated or down-regulated in the GO term. The inner circle has a double function: The height of the bar indicates the negative $\log _{10}$ adjusted $P$-value (the taller, the more significant), and the color of the bar represents the $z$-score. $(I, J)$ IPA canonical pathways analysis $(z$-score $>1.5, P<0.05$ for all; $I)$ and predicted regulators $(J)$ for the same comparison. 
confirmed by the expression of various subcluster signature genes such as Rrm2 (Hammond 2a), Ube2c (Hammond 2b), Cenpa (Hammond 2c), and Spp1 (Hammond 4) (Supplemental Fig. S2L-Q). Arg1, a marker for the fifth subcluster (Hammond 1), could not be used to define a specific subpopulation (Supplemental Fig. S2L). These findings were further confirmed when we performed a gene marker analysis to look for potential markers for each subcluster in the aggregated data set (Supplemental Fig. S4). The resulting heat map (Supplemental Fig. S4A) showed that typical gene markers could be found for most of the subclusters. In some of them, such as subcluster 8 , the expression of the marker genes was very distinct, with some of the genes also belonging to the typical BAM signature genes (Supplemental Fig. S4A,B). Furthermore, subclusters 3 and 9 showed similar expression of marker genes such as Spp1, Gpnmb, Igf1, and Lgals3 (Supplemental Fig. S4A,B), suggesting that these two subclusters, especially subcluster 3 , were similar to the main parenchymal microglia subcluster 4 at PND4/5 as suggested by Hammond et al. (2019) (Supplemental Fig. S2P,Q).

Examination of the frequencies of the two data sets in each subpopulation showed that subcluster 8 was the major meningeal microglia-like cell subcluster (with gene expression signatures that were distinct from the parenchymal microglia) (Fig. 4F; Supplemental Fig. S2L-P), indicating that the microglia-like cells obtained in the current study were not contaminants from the brain parenchyma.

We then further explored the differences between meningeal and parenchymal microglia focusing on subcluster 8. There were 486 differently expressed genes between the two data sets (Fig. 4G; Supplemental Data 2), and these were mainly involved in metabolic processes, such as mitochondrial and ribosomal genes (Supplemental Table S7). In addition, GO term enrichment showed increased expression of genes involved in biological processes such as myeloid cell differentiation, homeostasis, and stress response (Fig. 4H; Supplemental Data 3). Using IPA, increased metabolism (glycolysis and oxidative phosphorylation), immune-related pathways (Th1 pathway) (Fig. 4I), and regulators involved in cell viability and migration of immune cells (Runx1 and Csf1) (Fig. 4J) were found. Runx1 is a key regulator of myeloid cell proliferation and differentiation (Zusso et al. 2012), while Csf1 is fundamental for microglial maintenance in the white matter (EasleyNeal et al. 2019). Therefore, meningeal subcluster 8 seemed to be more involved in myeloid cell differentiation compared with the parenchymal counterpart and may also be involved in white matter development.

To obtain a more comprehensive overview of the functions of the different meningeal microglia-like cell subclusters, we next performed a comparative analysis where the gene expression signature of each subcluster was compared with all others using the same annotation used for identifying the subpopulations in the aggregation analysis (Fig. 5A; Supplemental Data 4). Some microglialike cells expressed typical BAM markers such as Mrc1 and Lyve1 and general signature genes for MHCII low BAMs, and after reclustering we again found that subclus- ter 8 specifically expressed these markers (Supplemental Figs. S2A-C, S4A,B). It has been previously reported that at embryonic day (E) 14.5 there is a parenchymal microglial population that shows BAM features (Hammond et al. 2019). This E14.5 subcluster (subcluster 6 at E14.5 in Hammond et al.) featured an expression signature including $M s 4 a 7, C c r 1$, and $M s 46 c$, genes that were also predominantly expressed in subcluster 8 (Supplemental Fig. $\mathrm{S} 2 \mathrm{R}, \mathrm{S})$. This may indicate that the neonatal meningeal microglia-like population identified in the current study represents a distinct kind of microglia-like cell population with BAM features that is present in the meninges at a later age than previously found in the parenchyma.

We thus focused on meningeal subcluster 8 for its distinct features and on subcluster 3 due to its similarity to the main parenchyma Spp1-enriched microglia subcluster found in PND4/5 mice. Among the most significantly upregulated genes in subcluster 8 were $P f 4$ and $D a b 2$, which are involved in chemoattraction and adhesion processes (Supplemental Fig. S4; Supplemental Table S8). Similarly, subcluster 8 was highly enriched in genes related to immune response, defense response, and stress response (Fig. 5B; Supplemental Data 3). These results were confirmed by IPA (Fig. 5C,D), and among the top up-regulated pathways we observed IL-8 signaling, which is involved in the immune response in microglial cells (Flynn et al. 2003). Several immune-related functions like chemotaxis and immune response of phagocytes and activation of myeloid cells were predicted to be up-regulated by IFN- $\gamma$, which is known to boost immune response and metabolism in microglia (Fig. 5D; Ritprajak and Azuma 2015; Kroczynska et al. 2016; Baik et al. 2019).

In subcluster 3, the top 20 up-regulated genes were mostly involved in immune response such as $S p p 1$, Lgals3, and Csf1, as well as genes with metabolic functions like Lpl and Pgam1 (Supplemental Table S9). GO term enrichment showed that biological processes related to iron transport, leukocyte chemotaxis, and myeloid leukocyte migration were enriched, in contrast to cell proliferation and defense response processes (Fig. 5E; Supplemental Data 3). IPA predicted that subcluster 3 had upregulated glycolysis and gluconeogenesis, while immune-related pathways were down-regulated (Fig. 5F).

Furthermore, developmental white matter-associated genes (Itgax, Igf1, Spp1, Cxc112, Gpnmb, Dkk2, Cd28, Csf1, Fabp5, Cc15, and C1qa) and disease-associated genes (Itgax, Apoe, Axl, Trem2, Lpl, Cst7, Csf1, Cd9, Ccl6, and Timp2) (Lloyd and Miron 2019) were mostly expressed in subcluster 3 , similar to the major parenchymal microglia at PND4/5 and PND7 (Hammond et al. 2019; Li et al. 2019), suggesting that a minor part of the meningeal microglia-like cells might not only be involved in immune cell responses, but also support oligodendrocyte and white matter development (Fig. 5G,H).

Overall, the other microglia-like cell subclusters showed differential regulation of biological processes such as immune response, cell cycle, metabolic, and neuronal functions when the differentially expressed genes were analyzed for GO term enrichment (Supplemental Fig. S5-S7; Supplemental Data 3). Subcluster 0 showed a 

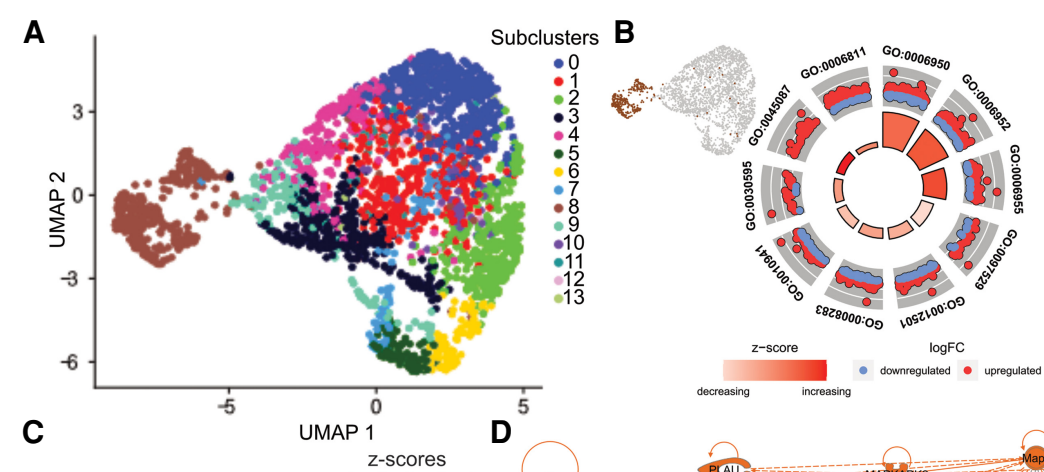

\begin{tabular}{|l|l|c|l|}
\hline ID & GO Term & Z-score & Adj_p-value \\
\hline GO:0006950 & response to stress & 4.423 & $2.87 \mathrm{e}-18$ \\
\hline GO:0006952 & defense response & 4.90 & $6.15 \mathrm{e}-17$ \\
\hline GO:0006955 & immune response & 5.18 & $1.93 \mathrm{e}-13$ \\
\hline GO:0097529 & myeloid leukocyte migration & 1.13 & $4.67 \mathrm{e}-09$ \\
\hline GO:0012501 & programmed cell death & 2.42 & $1.85 \mathrm{e}-08$ \\
\hline GO:0008283 & cell population proliferation & 2.8 & $5.98 \mathrm{e}-08$ \\
\hline GO:0010941 & regulation of cell death & 1.92 & $7.51 \mathrm{e}-08$ \\
\hline GO:0030595 leukocyte chemotaxis & 3 & $2.99 \mathrm{e}-07$ \\
\hline GO:0045087 & innate immune response & 6 & $3.78 \mathrm{e}-07$ \\
\hline GO:0006811 ion transport & 3.58 & $6.85 \mathrm{e}-06$ \\
\hline
\end{tabular}
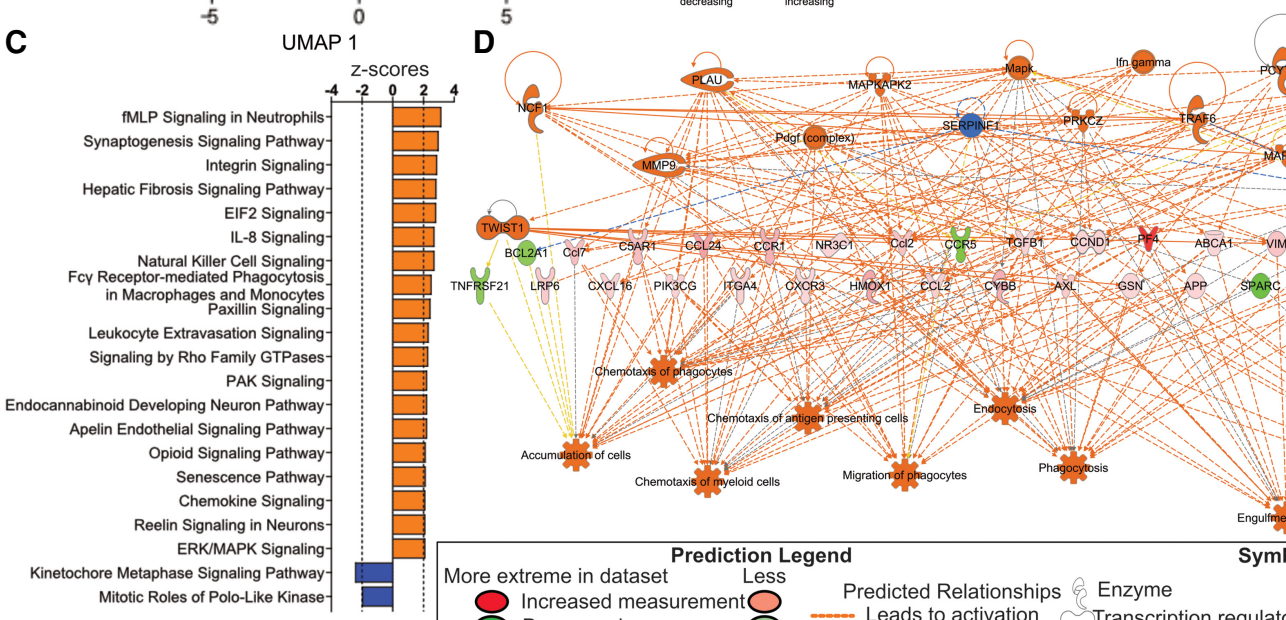
reduced biological function in immune response, while for subclusters 10,11, and 12 immune-related processes were enriched. These results reveal the considerable heterogeneity regarding immune response, indicating that some subclusters of microglia-like cells may have major biological functions other than taking part in the immune response, or perhaps a different rate of maturation of such functions for meningeal microglia-like cells. Another example of regulation of biological processes is cell cycle regulation, and subclusters 5, 6, and 7 had increased expression of cell cycle-related genes, suggesting that they are probably actively dividing cells and thus more immature, while similar processes were decreased in subclusters 1 and 4, perhaps indicating that they are cells with less proliferation potential. Subcluster 9 showed an enrichment in metabolic processes (Supplemental Fig. S6D), similar to subcluster 3 (Fig. 5F), further confirming that these two subclusters have a similar biological function profile as the main parenchymal microglia subcluster 4 at PND4/5 as suggested by in Hammond et al. (2019) In addition, subcluster 2 also showed enriched proliferative processes as seen previously for microglia in the first 2 wk of life (Nikodemova et al. 2015), and subcluster 13 seems to be involved in neurogenesis and nervous system development. Taken together, these results demonstrate the extensive heterogeneity and developmental trajectories of the different meningeal microglia-like cell populations during maturation.

Neutrophils, ILCs, T cells, and B cells are a minor fraction of the resident meningeal leukocytes in neonatal mice

We identified neutrophils, ILCs, T cells, and B cells in the neonatal mouse meninges, which agreed with previous findings (Albertsson et al. 2018; Nazmi et al. 2018; Zelco et al. 2020). These clusters were much smaller than BAM and microglia-like cell populations and contained only a few hundred cells (Fig. 1A,E; Supplemental Table S2).

Neutrophil populations are heterogeneous, with subdivisions based on the maturation state, which is strictly connected to the tissue in which they are found, with immature neutrophils typically found in the bone marrow, while mature cells can be found in peripheral blood and other tissues. These two subpopulations have been defined as stage I and stage II of differentiation /Giladi et al. 2018). Among others, typical genes for stage I neutrophils are S100a8 and S100a9, which were found among the signature genes for this cluster (Fig. 1F) and were homogeneously expressed at high levels in almost the entire neutrophil population (Supplemental Fig. S8A,B). Other genes for immature neutrophils, such as Lrg1 and Plscr1, were only expressed in a portion of the cells in this cluster (Supplemental Fig. S8C,D) indicating that neonatal meningeal neutrophils share both similarities and differences with peripheral immature neutrophils.

To analyze $\mathrm{T}$ cells and ILCs, we defined $\mathrm{T}$ cells as $C D 3 e^{+}$and ILCs as CD3e (Supplemental Fig. S8E). The ILC population was mainly composed of ILC2 (Gata3 ${ }^{\text {hi }}$, 84 cells) and ILC3 (Rorchi, 74 cells) subtypes, while ILC1s (Tbx21 ${ }^{h i}$, 25 cells) were only present in small num- bers (Supplemental Fig. S8F,G). Interestingly, we identified an unknown fourth subset of ILCs (57 cells) that expressed Gata3 at a low level (Supplemental Fig. S8G).

T cells (201 cells) (Supplemental Fig. S9A) were composed of $\gamma \delta$ T cells (subcluster 1, 62 cells), a $\beta$ T cells mainly expressing $C d 4$ and TCR $\beta$ (subcluster 0,118 cells), and a small subcluster 2 with likely a mix of T cells (21 cells) (Supplemental Fig. S9B). $\gamma \delta \mathrm{T}$ cells showed high expression levels of Tcry-V6 and Tcry-C1 as well as Th-17-like features (Supplemental Fig. S9B), which agreed with previous findings (Ribeiro et al. 2019). We also found some proliferating $\mathrm{T}$ cells, as shown by the expression of cell cycle genes (Supplemental Fig. S9C). To define the clonality of $\mathrm{T}$ - and B-cell receptors, we paired gene expression with immune profiling analysis. We detected Tcr $\alpha$ and Tcr $\beta$, but could not see any specific clone (Supplemental Fig. S9D-F) likely due to the low number of cells.

In addition, we observed a population of B cells (304 cells) (Supplemental Fig. S10A) with typical marker gene expression (Supplemental Fig. S10B), among which 6\% (21 cells) were identified as B1a cells using markers from a previous report (Tanabe and Yamashita 2018). Overall, the B-cell subclusters did not align with any of the known subtypes of mature B cells. Intriguingly, some B cells exhibited high expression of Mki67 (Supplemental Fig. S10B) and seemed to be proliferating (Supplemental Fig. S10C) but lacked Aicda, which is associated with the activation of mature B cells and germinal centre formation. Others have recently presented similar data from the spinal cord and brain meninges of adult mice, and they suggested that the CNS may act as a lymphopoietic niche (Brioschi et al. 2021; Cohen et al. 2021). The fact that we found proliferating B cells in early neonatal mouse meninges support this notion. The presence of early developing B cells may allow for counterselection of cells that are reactive against brain antigens, contribute to CNS development, and take part in disease pathology. Immune profiling analysis showed that B cells expressed immunoglobulin-L (Ig-L), Ig-K, and Ig-H, but no specific pattern of Ig expression was observed. Microglia-like cells also expressed Ig-H, confirming that microglia are capable of forming phagocyte complexes of IgG and Staphylococcus aureus through Fc receptors (Supplemental Fig. S10D-G; Quan et al. 2009).

\section{Neutrophils were the most affected population at $6 \mathrm{~h}$ after $\mathrm{HI}$}

We next investigated whether any of the meningeal leukocyte populations were affected $6 \mathrm{~h}$ after $\mathrm{HI}$ (Fig. 6B-D). The neutrophil cluster was the only population to be affected by $\mathrm{HI}$ both in terms of cell frequency-with a threefold-increase-and in terms of gene expression (Fig. 6C,D; Supplemental Data 5). Immunohistochemical staining of neutrophils using lymphocyte antigen 6 complex locus G6D (Ly6g) confirmed an increase in neutrophil density in the meninges $6 \mathrm{~h}$ after $\mathrm{HI}$ compared with naïve meninges, which was confirmed to be a neutrophil marker as it was coexpressed with myeloperoxidase (MPO), a classic neutrophil marker (Fig. 6E-G). Among the top 20 
A

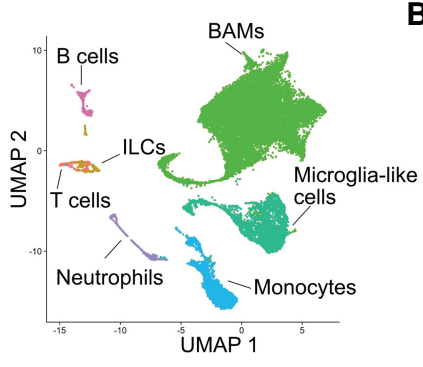

E

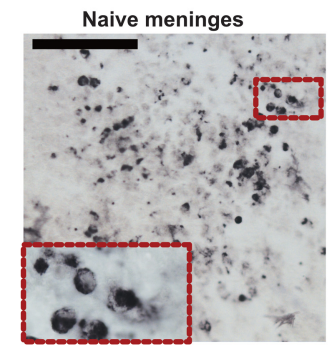

B

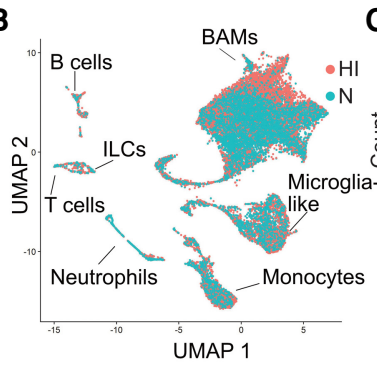

C

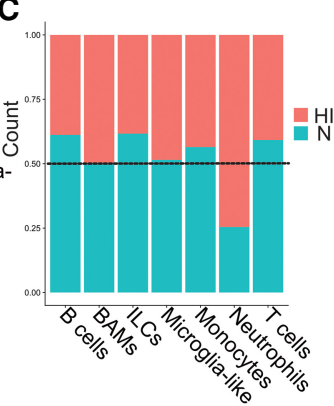

D

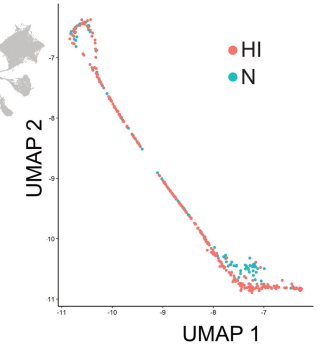

H

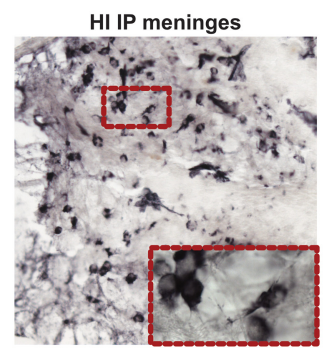

F

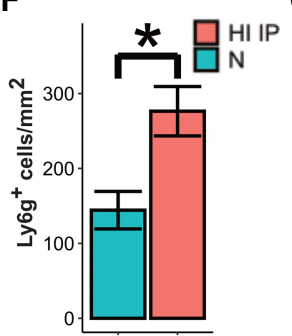

G

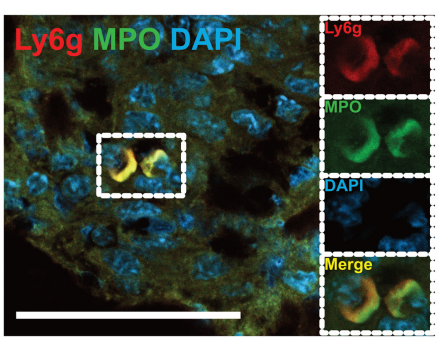

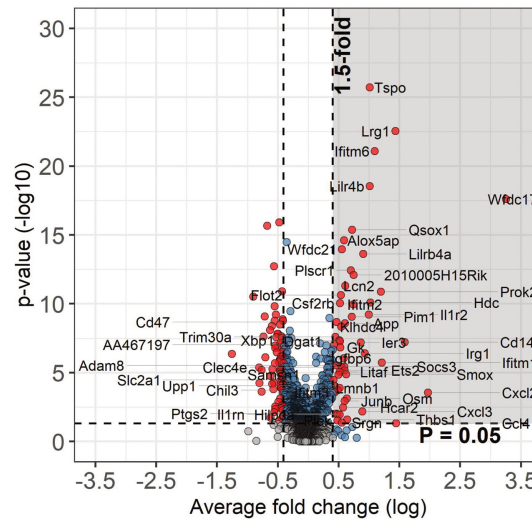

I

cat - Intermed • Non-sign - Sign

J

z-scores
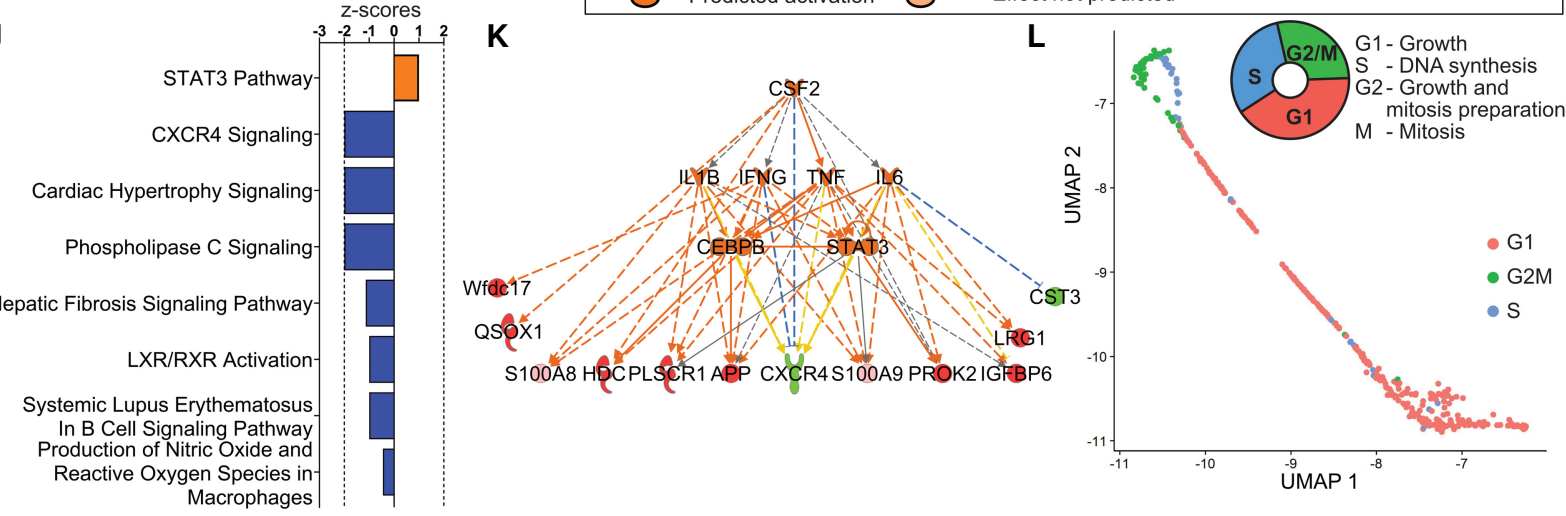

Figure 6. Neutrophils are increased at $6 \mathrm{~h}$ after HI. $(A-C)$ The cell frequency changes in each cluster of cells between naive and $\mathrm{HI}$ animals, shown as UMAP representing all clusters $(A)$, in $\mathrm{HI}$ or naïve samples $(B)$, and as a stacked bar chart $(C)$. (D) Representation of $\mathrm{HI}$ and naïve neutrophils. $(E, F)$ Immunohistochemistry staining of $\mathrm{Ly}_{6 \mathrm{~g}}{ }^{+}$neutrophils (scale bar, $\left.100 \mu \mathrm{m} ; E\right)$, and the density of $\mathrm{Ly} \mathrm{g}^{+}$neutrophils in meninges of naïve and $\mathrm{HI}$ mice $(F)$. $(G)$ Immunofluorescent double staining shows $\mathrm{Ly} 6 \mathrm{~g}^{+} \mathrm{MPO}^{+}$neutrophils in the mouse meninges. Scale bar, $50 \mu \mathrm{m}$. $(H)$ Volcano plot presenting differentially expressed genes between $\mathrm{HI}$ versus naïve mice. Genes in the gray area have fold change $>1.5$ and $P<0.05$. (I) GO term analysis using gProfiler. The inner circle has a double function: The height of the bar indicates the negative $\log _{10}$ adjusted $P$-value (the taller, the more significant), and the color of the bar represents the $z$-score. $(J, K)$ IPA of canonical pathways $(J)$ and predicted upstream regulator analysis $(K)$. $(L)$ Seurat cell cycle analysis for the neutrophil cluster. (IP) Ipsilateral. $\left(^{*}\right) P<$ 0.05 (unpaired $t$-test). 
significantly up-regulated genes (Fig. 6H; Supplemental Table S10) we found Tspo, which is involved in neutrophil inflammatory response (Kupa et al. 2017). Furthermore, we identified $\operatorname{Lrg} 1$ and Plscr1, genes with essential roles in neutrophil maturation, inflammatory responses, and granulocyte expansion (Frasch et al. 2004; Chen et al. 2011; Druhan et al. 2017). The increased neutrophil frequency could thus be explained by the up-regulation of Plscr1 after HI. Further analysis revealed enriched biological processes such as increased cellular response to chemical stimulus and response to cytokines (Fig. 6I; Supplemental Data 3). IPA confirmed these results with a positive $z$-score for the Stat3 pathway (Fig. 6J), which is involved in emergency granulopoiesis and triggers increased numbers of neutrophils by release from the bone marrow. Cxcr4 and phospholipase C signaling were down-regulated after HI (Fig. 6J). Csf2 was also predicted to be one of the upstream regulators, directly and indirectly affecting the expression of some of the top 20 genes, for example, through Stat 3 and Cebpb (Fig. 6K). These results indicate that the increased neutrophil frequency at this time point after HI might be due to increased cell division and not to infiltration of these cells from the blood.

To further confirm these results, we analyzed genes involved in the cell cycle. We scored the neutrophils based on cell cycle marker genes and classified them in terms of cell cycle phase as S, G1, or G2M (Fig. 6L). Most neutrophils were in G1 phase, where the cell synthesizes mRNA and proteins in preparation for mitosis. Of note, the majority of the neutrophils in G1 were those belonging to $\mathrm{HI}$ samples, implying that the insult stimulated neutrophil division and growth at $6 \mathrm{~h}$ after $\mathrm{HI}$.

In summary, at this early time $\mathrm{HI}$ triggers a signaling cascade that induces neutrophil expansion as part of the inflammatory process in response to tissue injury in the brain parenchyma, potentially through mechanisms such as emergency granulopoiesis.

\section{$B A M s$ and monocytes showed changes at $6 \mathrm{~h}$ after $H I$}

We next analyzed the BAM and monocyte response to $\mathrm{HI}$. These populations showed differences in gene expression without significant differences in cell frequency between $\mathrm{HI}$ and naïve samples (Supplemental Fig. S11A). A total of 1820 differentially expressed genes were found in BAMs after $\mathrm{HI}$, with 13 of them having a fold change $>1.5$ (Supplemental Fig. S11B; Supplemental Table S11; Supplemental Data 5). The GO analysis showed down-regulation of cellular localization and up-regulation of cellular metabolic processes (Supplemental Fig. S11C; Supplemental Data 3). IPA revealed up-regulation of RhoGD1 signaling, glycolysis, and PTEN signaling and down-regulation of pathways involved in actin cytoskeleton, IL-8 signaling, and phospholipase C signaling (Supplemental Fig. S11D). PTEN signaling mediates cellular responses in developing neurons, and its inhibition has been shown to be protective in cultured rat neurons under oxygen-glucose deprivation (Zhao et al. 2013). The regulator effect analysis suggested up-regulation of adhesion of blood cells and engulfment of phagocytoses, while axon genesis, cell outgrowth, growth of neurites, and proliferation of neuronal cells were all down-regulated. Several regulators were predicted to trigger the up-regulation and down-regulation of many genes, therefore increasing immune response, binding, and activation of leukocytes and phagocytes (Supplemental Fig. S11E). Among the regulators, we identified Arg1, which is predicted to lead to the down-regulation of processes like neuritogenesis, sprouting, and branching of cells through Igf1 and other genes (Supplemental Fig. S11F).

The uniform manifold approximation and projection (UMAP) plot indicated differences between $\mathrm{HI}$ and naïve BAMs (Fig. 7A). Therefore, we performed a detailed analysis of the differences between $\mathrm{HI}$ and naïve BAM subclusters (Fig. 7B,C). Indeed, the frequency analysis for all 8 subclusters of BAMs showed increased frequencies of subclusters 2 and 4 and decreased frequencies of subclusters $1,5,6$, and 7 after HI, though many comparisons resulted in low numbers of either differentially expressed genes (Supplemental Data 5) or cells (subcluster 6) (Fig. 7D). Gene marker analyses showed that the BAM subclusters that were decreased after $\mathrm{HI}(1,5,6$, and 7$)$ were characterized by the expression of genes involved in neuronal development and cell migration, while immune responserelated processes were diminished (Fig. 7E; Supplemental Figs. S12, S13; Supplemental Data 3, 6), indicating their possible role in CNS development rather than in immune response. The HI insult disrupts mitochondrial respiration and can lead to impaired brain development (Gopagondanahalli et al. 2016), thus making it plausible that these BAM subclusters, especially subcluster 6 , are more vulnerable and easily affected by the $\mathrm{HI}$ insult. Together this may indicate that $\mathrm{HI}$ insult affects the immature brain not only by directly causing neuronal cell death, but also by impacting on brain development indirectly by influencing the activity of meningeal BAMs in metabolism, cell proliferation, and neuron development. In contrast, BAM subclusters 2 and 4, which were increased the most after $\mathrm{HI}$, were strongly enriched in immune-related biological processes, with subcluster 2 also showing enrichment of cell death-related and migration-related processes (Fig. 7E; Supplemental Figs. S12, S13; Supplemental Data 3).

Next, we focused on BAM subcluster 2 because this was the subcluster most affected by HI (Fig. 7D). A total of 437 differentially expressed genes were found between $\mathrm{HI}$ and naïve mice (Fig. 7F; Supplemental Table S12). GO term enrichment highlighted down-regulation of immune-related processes in HI compared with naïve mice (Fig. 7G; Supplemental Data 3), which was confirmed by IPA (Fig. 7H,I). There was also an increase in metabolic pathways similar to what was seen in the total population of BAMs. Regulators such as $P f 4$, a typical BAM gene important for chemoattraction and cell adhesion, were predicted to downregulate functions such as development of the hematopoietic system, which is important for the immune response after preterm brain injury (Fig. 7I). All together, these results suggest that meningeal BAMs might contribute to the immune response after $\mathrm{HI}$ as well as the HI- 
Zelco et al.

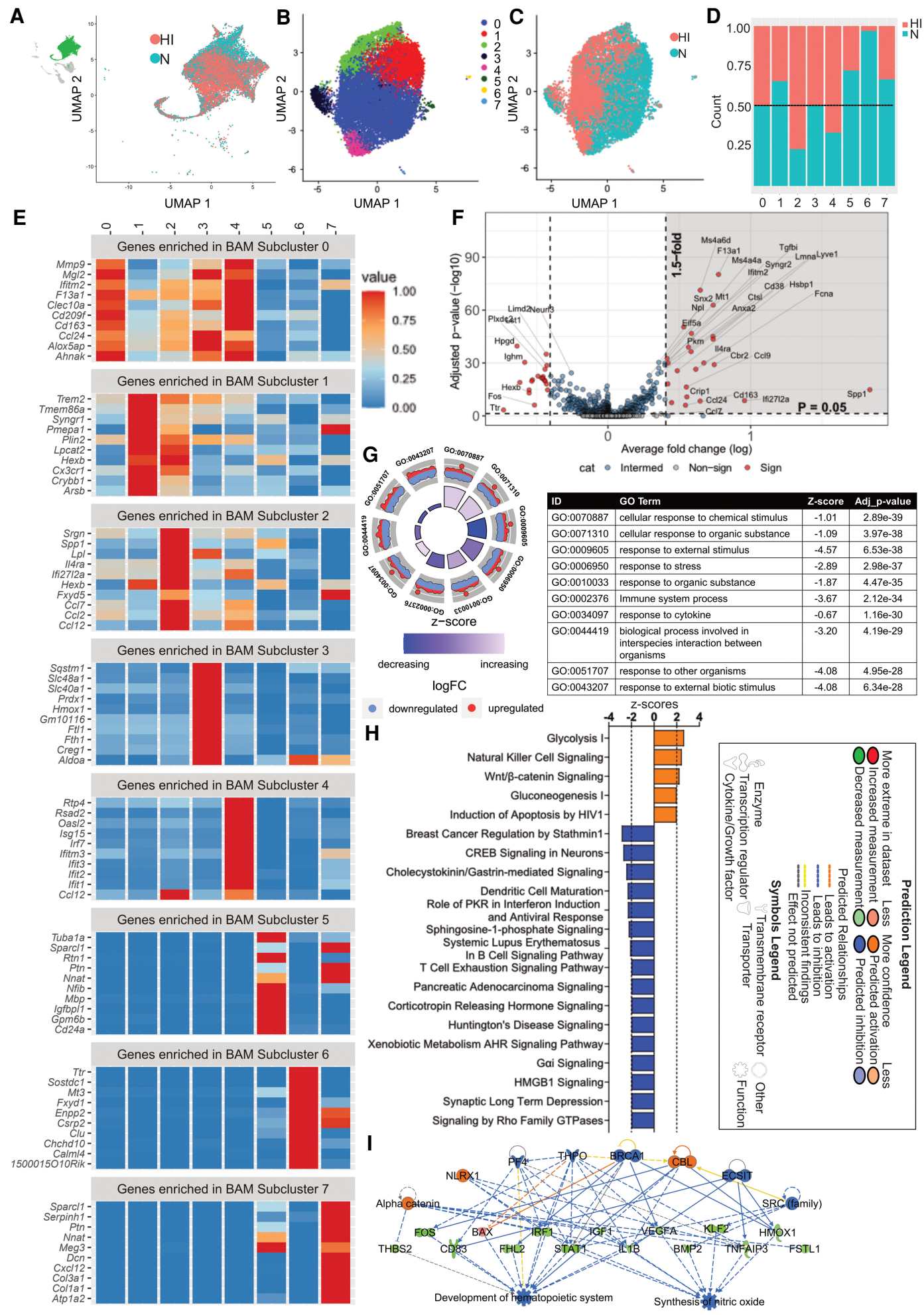

Figure 7. BAM subcluster 2 is the most affected after HI, with down-regulation of immune response-related processes at $6 \mathrm{~h}$ after $\mathrm{HI}$. $(A)$ UMAP plot showing the BAM population colored as cells from $\mathrm{HI}$ or naïve mice. $(B-D)$ BAMs subclusters $(B)$ showing as cells from naïve or $\mathrm{HI}$ mice as a UMAP plot $(C)$ or a frequency stacked bar chart $(D)$. $(E)$ Heat map showing the signature gene expression for each BAM subcluster from $B)$. $(F, G)$ Differentially expressed gene analysis comparing BAM subcluster 2 in $\mathrm{HI}$ and naïve mice as a volcano plot (gray quadrant; $P<0.05$ and fold change $>1.5 ; F)$, and GO term analysis $(G) .(H, I)$ IPA for canonical pathways $(H)$ and regulators $(I)$ in HI versus naïve BAMs. $(G)$ For the GO term analysis, the inner circle has a double function: The height of the bar indicates the negative $\log _{10}$ adjusted $P$ value (the taller, the more significant), and the color of the bar represents the $z$-score. $(H)$ For the IPA canonical pathways analysis, only the pathways with $z$-score $>1.5$ are shown. 
triggered disturbances in neuronal development in the parenchyma.

Monocyte numbers did not differ between $\mathrm{HI}$ and naïve mice (Supplemental Fig. S11G). There were 1720 differentially expressed genes between the two conditions, with 16 of them having a fold change $>1.5$ (Supplemental Fig. $\mathrm{S} 11 \mathrm{H}$; Supplemental Table S13; Supplemental Data 5). Further analysis showed biological processes involved in metabolic functions and down-regulation of immune system processes in general (Supplemental Fig. S11I; Supplemental Data 3). Interestingly, IPA instead showed specific immune pathways to be activated, such as the Th17 pathway (Supplemental Fig. S11J). Ccr2 and Il-5 were predicted to trigger the increased expression of S100a8 and Soc3, which were among the top up-regulated genes (Supplemental Fig. S11K).

To summarize, neonatal meningeal BAMs and monocytes reacted to $\mathrm{HI}$ at this early stage and induced an inflammatory gene response, and BAMs also trigged the down-regulation of functions connected to the proliferation and growth of neurons.

Regarding the other clusters, microglia-like cells did not show any obvious differences between $\mathrm{HI}$ and naïve mice at the global level or for subclusters. However, we detected significant gene expression differences when analyzing the microglial white matter and disease-associated genes (Supplemental Fig. S14). The T-cell, ILC, and B-cell populations were not affected by $\mathrm{HI}$ at this time point (Supplemental Data 5).

\section{Discussion}

In the current study, we have for the first time characterized the meningeal leukocyte landscape at the single-cell level in PND4 neonatal mice, established the developmental differences between neonatal and adult meningeal BAMs, and analyzed in detail the meninges to show the early leukocyte response after neonatal HI.

We found that the majority of meningeal immune cells were BAMs, microglia-like cells, and monocytes, whereas other cell populations, such as neutrophils, T cells, B cells, and ILCs, were present in smaller numbers. This is in agreement with previous studies in which all observed cell populations were described in the meninges of mouse embryos (Tanabe and Yamashita 2018; Utz et al. 2020), neonatal mouse pups (Albertsson et al. 2018; Nazmi et al. 2018; Tanabe and Yamashita 2018; Zelco et al. 2020), and adult mice (Gadani et al. 2017; Mrdjen et al. 2018; Van Hove et al. 2019|. BAMs are newly defined macrophages found in the meninges, choroid plexus, and perivascular spaces of brain vessels, and only a few studies have characterized the meningeal BAM populations at different developmental ages (Mrdjen et al. 2018; Van Hove et al. 2019; Utz et al. 2020). One of the challenges of studying BAMs is that they share gene expression with microglia and brain macrophages and, to a lesser extent, with monocytes, as corroborated by the trajectory analyses in the current study. However, we confirmed that a combination of markers for immunofluorescence staining can be used to identify meningeal BAMs in neonatal mice, in agreement with previous reports at other developmental stages (Mrdjen et al. 2018; Van Hove et al. 2019; Utz et al. 2020).

The current study is the first to establish the developmental differences between neonatal and adult meningeal BAMs. We found increased expression of cytoskeleton-associated genes in neonatal BAMs compared with meningeal BAMs in adult mice, similar to what was found in neonatal peritoneal macrophages (Winterberg et al. 2015). Basic macrophage functions, like phagocytosis, are already present at the neonatal stage (Speer et al. 1988), but compared with adults neonatal BAMs had reduced expression of genes related to antigen-presenting cell response and recruitment of cells as also shown in the current study. This suggests that although some primary functions are already present at this early stage of life, neonatal BAMs are functionally immature and possibly unable to generate a full immune response.

Sall1 was used to specifically identify microglia because it is not expressed by meningeal BAMs, and here we show that Sall1 expression was indeed predominant in the newly identified meningeal microglia-like cell cluster at this developmental age. In total, our aggregation analysis of parenchymal microglia identified 14 subclusters of microglia, with subcluster 8 being a BAM-like subcluster and subcluster 3 representing the major age-identical parenchymal microglial subpopulation (Hammond et al. 2019). Analysis of differential gene expression between meningeal and parenchymal subcluster 8 highlighted an increase in mitochondrial and immune-related function, indicating that this meningeal subpopulation might quickly respond to insults. Further analyses showed that meningeal subcluster 8 also had high expression of genes such as Pf4, Dab2, and Fcna. Although Pf4 and Dab2 have been reported in embryonic microglia, all three genes were also found to be expressed in BAMs instead of microglia (Van Hove et al. 2019; Utz et al. 2020), with Fcna being a specific marker for MHCII low BAMs (Van Hove et al. 2019). Indeed, we also observed low expression of MHCII genes in part of the microglia-like cell cluster, and these overlapped with Mrc1 and Lyve1 in microglia-like cells. These findings indicate a possible new subtype of meningeal myeloid cells that have characteristics of both microglia and BAMs and that express both the microgliaspecific marker Sall1, as has been described in the choroid plexus (Van Hove et al. 2019), and BAM markers. It is unlikely that these cells with mixed phenotypes represent a transitional or developmental stage between the microglia and macrophages because the parenchymal microglia and BAMs separate early in development and can be identified as two individual lineages in terms of transcription, phenotype, location, and cytokine requirement (Utz et al. 2020).

Compared with all other meningeal subpopulations, microglia-like cell subcluster 3 and 9 expressed higher levels of Spp1, Lgals3, Gpnmb, and Igf1, which are markers for the major parenchymal microglia subpopulation at PND4/5 (Hammond et al. 2019). These Spp1-enriched microglia mainly appear transiently in the developing 
white matter in early postnatal age (PND4-7) and constitute the major parenchymal microglial population at PND4/5 but are barely found at any other developmental ages (Hammond et al. 2019; Li et al. 2019). The fact that this microglia-like cell subpopulation was found in the meninges at an early postnatal age might suggest again that meningeal immune cells participate in brain development. Indeed, factors such as retinoic acid released from the meninges can influence cortical neuronal development (Siegenthaler et al. 2009), and a cascade of morphogenic signals initiated by the meninges regulates corpus callosum development (Choe et al. 2012). Also, B1a cells, a subtype of $\mathrm{B}$ cells resident in the meninges, are involved in oligodendrocyte development through IgM-Fca $/ \mu \mathrm{R}$ signaling (Tanabe and Yamashita 2018). Further research is needed to determine whether Spp1-menigeal microglialike cells have a role in white matter development.

Six hours is generally considered to be the limit of the therapeutic window after HI because this is when the initial injury, caused mainly by the accumulation of reactive oxygen species due to mitochondrial failure, becomes irreversible and leads to neuronal death. Previously, we found that $\gamma \delta$ T cells and ILC2s are increased in the meninges and in the brain parenchyma at $6 \mathrm{~h}$ after $\mathrm{HI}$ in a preterm brain injury mouse model (Albertsson et al. 2018; Zelco et al. 2020). In the current study, we found that most of the meningeal immune cell populations were not affected. Neutrophils were the main subpopulation to be affected by $\mathrm{HI}$ at $6 \mathrm{~h}$, with both increased numbers and altered gene expression profiles. Neutrophils are the main leukocyte subtype present in the first week after birth in mice, and they are early responders (within $6 \mathrm{~h}$ ) after insult in multiple animal models of brain injury, including in neonates. Emergency granulopoiesis is a phenomenon that causes the release of immature neutrophils from the bone marrow in order to respond to pathogens or to tissue damage. Cebpb, one of the upstream factors identified in the IPA, is essential for initiating emergency granulopoiesis by cell cycle acceleration through c-Myc (Hirai et al. 2006). The switch between regular and emergency granulopoiesis is mediated mainly through the Stat 3 pathway (Panopoulos et al. 2006), which was identified in the IPA of canonical pathways. This is further supported by the down-regulation of the Cxcr4 pathway and the increase in cell growth observed in cell cycle analysis. Cxcr4 has been shown to be involved in the retention of neutrophils either in bone marrow or at the site of injury (Yamada et al. 2011; De Filippo and Rankin 2018), and increased Csf2 expression results in a reduction of Cxcr4, similar to what we observed. Increased Csf2 and decreased Cxcr4 might indicate that neutrophils that are normally retained in the meninges expand by emergency granulopoiesis after $\mathrm{HI}$ and are mobilized to initiate the inflammatory response cascade.

Notably, in the current study sham controls were not included because previous studies showed no differences between sham and naïve controls (McRae et al. 1995). We cannot exclude the possibility that the anesthesia and the manipulation of the carotid artery may indeed cause subtle changes at the gene level; however, the re- ported major differences between the $\mathrm{HI}$ and naïve mice are robust and unlikely to be due to these experimental procedures.

In conclusion, our findings suggest a prominent role for neonatal mouse meningeal leukocytes in physiological processes and after preterm brain injury. The resident meningeal leukocytes are highly heterogeneous and tissue-specific, and neonatal BAMs share similarities but also feature pronounced differences compared with their adult counterparts in terms of phenotype and function. Strikingly, we identified a distinct meningeal cell population clearly expressing the molecular signature of microglia but different from the parenchymal microglia. These meningeal microglia-like cells may participate in brain development and help maintain CNS homeostasis. Furthermore, we highlighted the meninges as an important site of immune cell expansion and reactivity as part of the early immune response in the brain after injury. Finally, the meningeal leukocytes may take part in normal CNS development, and once affected by an insult they may interrupt normal neuronal and axonal development. Altogether, the present study provides novel insights into CNS physiology and offers an essential resource for comparative analyses and data exploration that facilitates a deeper understanding of the functional role of the meninges in brain development, health, and disease.

\begin{abstract}
Materials and methods
Experimental animals

C57/B16J (Charles River) mice were used for all experiments and were bred at the Experimental Biomedicine animal facility of the University of Gothenburg. The animal room has a 12-h light-dark cycle, and the mice had free access to chow $(\mathrm{B} \& \mathrm{~K})$ and water. All experiments were approved by the Regional Animal Ethical Committee of Gothenburg (58/2016 and 2042/18).
\end{abstract}

Single-cell RNA sequencing sample preparation and analysis

The 10X Genomics chromium single-cell 5' library and gel bead kit was used for high-throughput automated barcoding and library construction following the manufacturer's instructions (four runs, version 1, PN-1000014). The immune profiling V(D)J libraries were constructed using a single-cell $5^{\prime}$ library and gel gead kit and single-cell V(D)J enrichment kit (mouse T cells and B cells). After cDNA library preparation, the samples were analyzed on a NextSeq 500/550 and MiSeq (Illumina) at the Clinical Genomics Gothenburg, SciLife Laboratories. Detailed methods and analysis are in the Supplemental Material. The data were deposited in the Gene Expression Omnibus (GEO) database under accession number GSE173769.

Immunofluorescent and immunohistochemical staining

After transcardiac saline perfusion, the meninges of PND4 mice were peeled off from the brain, each hemisphere separately, and positioned on a glass slide. The staining was performed as described (Zelco et al. 2020) in the Supplemental Material.

See the Supplemental Material for additional Materials and Methods. 


\section{Competing interest statement}

V.M.L. is cofounder, CEO, and shareholder of HepaPredict $A B$, and cofounder and shareholder of PersoMedix AB. In addition, V.M.L. discloses consultancy work for EnginZyme AB. The authors have no additional financial interests.

\section{Acknowledgments}

We thank the Genomics and Bioinformatics Core Facility platforms at the Sahlgrenska Academy, University of Gothenburg, for performing the sequencing, CellRanger, and scRNA-seq bioinformatics analysis. We also acknowledge Alina Orozco for help with the bioinformatics analysis, Joakim Ek for help with IPA, Tetyana Chumak and Anna-Lena Leverin for help with the animal experiments (Department of Physiology, Sahlgrenska Academy, University of Gothenburg), and Kajsa Groustra for help with mouse breeding (Experimental Biomedicine, Sahlgrenska Academy, University of Gothenburg). This project was supported by the Swedish Research Council (2015-06276 and 2018-02682 to X.W., and 2019-01320 to H.H.), the Brain Foundation (FO2017-0102 to X.W.); grants from the Swedish state under the agreement between the Swedish government and the county councils, the ALF agreement (ALFGBG-429801 and ALFGBG-813291 to X.W., and ALFGBG-718591 to H.H.|n the National Natural Science Foundation of China (81771418 and U1704281 to X.W.), the Elisabet Bollan Foundation (2019 to A.Z.); and Herbert och Karin Jacobssons Stiftelse (2018 and 2020 to A.Z.).

Author contributions: X.W. and A.Z. conceptualized the study and wrote the manuscript. A.Z., X.W., C.M., and H.H. designed the experiments. P.S. and A.Z. performed the HI model. A.Z. and S.N. collected and prepared the samples for FACS. M.B., A.Z., and E.R.-F. performed the cell sorting. C.L.-F. and A.Z. performed library preparation for sequencing. A.Z. and G.N. performed the immunofluorescence and immunohistochemistry staining experiments. V.B., J.K.d.K., A.Z., and V.M.L. performed the bioinformatics analysis with intellectual contribution from X.W., F.C.P.H., and M.B. All authors contributed to data interpretation and critical revision of the manuscript.

\section{References}

Akiyama H, McGeer PL. 1990. Brain microglia constitutively express $\beta$-2 integrins. I Neuroimmunol 30: 81-93. doi:10.1016/ 0165-5728(90)90055-R

Albertsson A, Zhang X, Vontell R, Bi D, Bronson RT, Supramaniam V, Baburamani AA, Hua S, Nazmi A, Cardell S, et al. 2018. $\gamma \delta$ T cells contribute to injury in the developing brain. Am J Pathol 188: 757-767. doi:10.1016/j.ajpath.2017.11.012

Alves de Lima K, Rustenhoven J, Da Mesquita S, Wall M, Salvador AF, Smirnov I, Martelossi Cebinelli G, Mamuladze T, Baker W, Papadopoulos Z, et al. 2020. Meningeal $\gamma \delta \mathrm{T}$ cells regulate anxiety-like behavior via IL-17a signaling in neurons. Nat Immunol 21: 1421-1429. doi:10.1038/s41590-020-0776-4

Back SA. 2017. White matter injury in the preterm infant: pathology and mechanisms. Acta Neuropathol 134: 331-349. doi:10 .1007/s00401-017-1718-6

Back SA, Luo NL, Borenstein NS, Levine JM, Volpe JJ, Kinney HC. 2001. Late oligodendrocyte progenitors coincide with the developmental window of vulnerability for human perinatal white matter injury. J Neurosci 21: 1302-1312. doi:10.1523/ JNEUROSCI.21-04-01302.2001

Baik SH, Kang S, Lee W, Choi H, Chung S, Kim JI, Mook-Jung I. 2019. A breakdown in metabolic reprogramming causes microglia dysfunction in Alzheimer's disease. Cell Metab 30: 493-507.e6. doi:10.1016/j.cmet.2019.06.005

Brioschi S, Wang W-L, Peng V, Wang M, Shchukina I, Greenberg ZJ, Bando JK, Jaeger N, Czepielewski RS, Swain A, et al. 2021. Heterogeneity of meningeal B cells reveals a lymphopoietic niche at the CNS borders. Science eabf9277. doi:10.1126/sci ence.abf 9277

Chen CW, Sowden M, Zhao Q, Wiedmer T, Sims PJ. 2011. Nuclear phospholipid scramblase 1 prolongs the mitotic expansion of granulocyte precursors during G-CSF-induced granulopoiesis. J Leukoc Biol 90: 221-233. doi:10.1189/jlb.0111006

Choe Y, Siegenthaler JA, Pleasure SJ. 2012. A cascade of morphogenic signaling initiated by the meninges controls corpus callosum formation. Neuron 73: 698-712. doi:10.1016/j.neuron .2011 .11 .036

Cohen M, Giladi A, Raposo C, Zada M, Li B, Ruckh J, Deczkowska A, Mohar B, Shechter R, Lichtenstein RG, et al. 2021. Meningeal lymphoid structures are activated under acute and chronic spinal cord pathologies. Life Sci Alliance 4: e202000907. doi:10.26508/1sa.202000907

De Filippo K, Rankin SM. 2018. CXCR4, the master regulator of neutrophil trafficking in homeostasis and disease. Eur J Clin Invest 48: e12949. doi:10.1111/eci.12949

de Kanter JK, Lijnzaad P, Candelli T, Margaritis T, Holstege F. 2019. CHETAH: a selective, hierarchical cell type identification method for single-cell RNA sequencing. Nucleic Acids Res 47: e95. doi:10.1093/nar/gkz543

DeSisto J, O'Rourke R, Jones HE, Pawlikowski B, Malek AD, Bonney S, Guimiot F, Jones KL, Siegenthaler JA. 2020. Single-cell transcriptomic analyses of the developing meninges reveal meningeal fibroblast diversity and function. Dev Cell 54: 43-59.e4. doi:10.1016/j.devcel.2020.06.009

Druhan LJ, Lance A, Li S, Price AE, Emerson JT, Baxter SA, Gerber JM, Avalos BR. 2017. Leucine rich $\alpha-2$ glycoprotein: a novel neutrophil granule protein and modulator of myelopoiesis. PLoS One 12: e0170261. doi:10.1371/journal.pone.0170261

Easley-Neal C, Foreman O, Sharma N, Zarrin AA, Weimer RM. 2019. CSF1R ligands IL-34 and CSF1 Are differentially required for microglia development and maintenance in white and gray matter brain regions. Front Immunol 10: 2199. doi:10.3389/fimmu.2019.02199

Flynn G, Maru S, Loughlin J, Romero IA, Male D. 2003. Regulation of chemokine receptor expression in human microglia and astrocytes. I Neuroimmunol 136: 84-93. doi:10.1016/ S0165-5728(03)00009-2

Frasch SC, Henson PM, Nagaosa K, Fessler MB, Borregaard N, Bratton DL. 2004. Phospholipid flip-flop and phospholipid scramblase 1 (PLSCR1) co-localize to uropod rafts in formylated Met-Leu-Phe-stimulated neutrophils. J Biol Chem 279: 17625-17633. doi:10.1074/jbc.M313414200

Gadani SP, Smirnov I, Smith AT, Overall CC, Kipnis J. 2017. Characterization of meningeal type 2 innate lymphocytes and their response to CNS injury. I Exp Med 214: 285-296. doi:10.1084/jem.20161982

Giladi A, Paul F, Herzog Y, Lubling Y, Weiner A, Yofe I, Jaitin D, Cabezas-Wallscheid N, Dress R, Ginhoux F, et al. 2018. Single-cell characterization of haematopoietic progenitors and their trajectories in homeostasis and perturbed haematopoiesis. Nat Cell Biol 20: 836-846. doi:10.1038/s41556-018-0121-4

Gopagondanahalli KR, Li J, Fahey MC, Hunt RW, Jenkin G, Miller SL, Malhotra A. 2016. Preterm hypoxic-ischemic encephalopathy. Front Pediatr 4: 114. doi:10.3389/fped.2016.00114

Hammond TR, Dufort C, Dissing-Olesen L, Giera S, Young A, Wysoker A, Walker AJ, Gergits F, Segel M, Nemesh J, et al. 2019. Single-cell RNA sequencing of microglia throughout 
the mouse lifespan and in the injured brain reveals complex cell-state changes. Immunity 50: 253-271.e6. doi:10.1016/j .immuni.2018.11.004

Herz J, Köster C, Crasmöller M, Abberger H, Hansen W, Felderhoff-Müser U, Bendix I. 2018. Peripheral T cell depletion by FTY720 exacerbates hypoxic-ischemic brain injury in neonatal mice. Front Immunol 9: 1696. doi:10.3389/fimmu.2018 .01696

Hirai H, Zhang P, Dayaram T, Hetherington CJ, Mizuno S, Imanishi J, Akashi $\mathrm{K}$, Tenen DG. 2006. C/EBP $\beta$ is required for 'emergency' granulopoiesis. Nat Immunol 7: 732-739. doi:10.1038/ni1354

Hurn PD, Subramanian S, Parker SM, Afentoulis ME, Kaler LJ, Vandenbark AA, Offner H. 2007. T- and B-cell-deficient mice with experimental stroke have reduced lesion size and inflammation. I Cereb Blood Flow Metab 27: 1798-1805. doi:10.1038/sj.jcbfm.9600482

Juul SE, Comstock BA, Wadhawan R, Mayock DE, Courtney SE, Robinson T, Ahmad KA, Bendel-Stenzel E, Baserga M, LaGamma EF, et al. 2020. A randomized trial of erythropoietin for neuroprotection in preterm infants. $N$ Engl $I$ Med 382: 233-243. doi:10.1056/NEJMoa1907423

Kroczynska B, Rafidi RL, Majchrzak-Kita B, Kosciuczuk EM, Blyth GT, Jemielity J, Warminska Z, Saleiro D, Mehrotra S, Arslan $\mathrm{AD}$, et al. 2016. Interferon $\gamma(\mathrm{IFN} \gamma)$ signaling via mechanistic target of rapamycin complex 2 (mTORC2) and regulatory effects in the generation of type II interferon biological responses. I Biol Chem 291: 2389-2396. doi:10.1074/jbc .M115.664995

Kupa LVK, Drewes CC, Barioni ED, Neves CL, Sampaio SC, Farsky SHP. 2017. Role of translocator $18 \mathrm{KDa}$ ligands in the activation of leukotriene B4 activated G-protein coupled receptor and toll like receptor-4 pathways in neutrophils. Front Pharmacol 8: 766. doi:10.3389/fphar.2017.00766

Li Q, Cheng Z, Zhou L, Darmanis S, Neff NF, Okamoto J, Gulati G, Bennett ML, Sun LO, Clarke LE, et al. 2019. Developmental heterogeneity of microglia and brain myeloid cells revealed by deep single-cell RNA sequencing. Neuron 101: 207223.e10. doi:10.1016/j.neuron.2018.12.006

Lloyd AF, Miron VE. 2019. The pro-remyelination properties of microglia in the central nervous system. Nat Rev Neurol 15: 447-458. doi:10.1038/s41582-019-0184-2

Mallard C, Vexler ZS. 2015. Modeling ischemia in the immature brain: how translational Are animal models? Stroke 46: 30063011. doi:10.1161/STROKEAHA.115.007776

McRae A, Gilland E, Bona E, Hagberg H. 1995. Microglia activation after neonatal hypoxia-ischemia. Developmental Brain Research 84: 245-252 . doi:10.1016/0165-3806(94)00177-2

Mrdjen D, Pavlovic A, Hartmann FJ, Schreiner B, Utz SG, Leung BP, Lelios I, Heppner FL, Kipnis J, Merkler D, et al. 2018. Highdimensional single-cell mapping of central nervous system immune cells reveals distinct myeloid subsets in health, aging, and disease. Immunity 48: 380-395.e6. doi:10.1016/j .immuni.2018.01.011

Nazmi A, Albertsson A, Rocha-Ferreira E, Zhang X, Vontell R, Zelco A, Rutherford M, Zhu C, Nilsson G, Mallard C, et al. 2018. Lymphocytes contribute to the pathophysiology of neonatal brain injury. Front Neurol 9: 159. doi:10.3389/fneur .2018.00159

Nikodemova M, Kimyon RS, De I, Small AL, Collier LS, Watters JJ. 2015. Microglial numbers attain adult levels after undergoing a rapid decrease in cell number in the third postnatal week. J Neuroimmunol 278: 280-288. doi:10.1016/j.jneuroim.2014 .11 .018
Panopoulos AD, Zhang L, Snow JW, Jones DM, Smith AM, El Kasmi KC, Liu F, Goldsmith MA, Link DC, Murray PJ, et al. 2006. STAT3 governs distinct pathways in emergency granulopoiesis and mature neutrophils. Blood 108: 3682-3690. doi:10.1182/blood-2006-02-003012

Quan Y, Möller T, Weinstein JR. 2009. Regulation of Fc $\gamma$ receptors and immunoglobulin G-mediated phagocytosis in mouse microglia. Neurosci Lett 464: 29-33. doi:10.1016/j.neulet .2009.08.013

Ribeiro M, Brigas HC, Temido-Ferreira M, Pousinha PA, Regen T, Santa C, Coelho JE, Marques-Morgado I, Valente CA, Omenetti $S$, et al. 2019. Meningeal $\gamma \delta$ T cell-derived IL-17 controls synaptic plasticity and short-term memory. Sci Immunol 4: eaay5199. doi:10.1126/sciimmunol.aay5199

Ritprajak P, Azuma M. 2015. Intrinsic and extrinsic control of expression of the immunoregulatory molecule PD-L1 in epithelial cells and squamous cell carcinoma. Oral Oncol 51: 221228. doi:10.1016/j.oraloncology.2014.11.014

Schafer DP, Lehrman EK, Kautzman AG, Koyama R, Mardinly AR, Yamasaki R, Ransohoff RM, Greenberg ME, Barres BA, Stevens B. 2012. Microglia sculpt postnatal neural circuits in an activity and complement-dependent manner. Neuron 74: 691-705. doi:10.1016/j.neuron.2012.03.026

Shichita T, Sugiyama Y, Ooboshi H, Sugimori H, Nakagawa R, Takada I, Iwaki T, Okada Y, Iida M, Cua DJ, et al. 2009. Pivotal role of cerebral interleukin-17-producing $\gamma \delta \mathrm{T}$ cells in the delayed phase of ischemic brain injury. Nat Med 15: 946-950. doi:10.1038/nm.1999

Shrestha N, Bahnan W, Wiley DJ, Barber G, Fields KA, Schesser K. 2012. Eukaryotic initiation factor 2 (eIF2) signaling regulates proinflammatory cytokine expression and bacterial invasion. J Biol Chem 287: 28738-28744. doi:10.1074/jbc.M112.375915

Siegenthaler JA, Ashique AM, Zarbalis K, Patterson KP, Hecht $\mathrm{JH}$, Kane MA, Folias AE, Choe Y, May SR, Kume T, et al. 2009. Retinoic acid from the meninges regulates cortical neuron generation. Cell 139: 597-609. doi:10.1016/j.cell .2009 .10 .004

Song J, Sun H, Xu F, Kang W, Gao L, Guo J, Zhang Y, Xia L, Wang X, Zhu C. 2016. Recombinant human erythropoietin improves neurological outcomes in very preterm infants. Ann Neurol 80: 24-34. doi:10.1002/ana.24677

Speer CP, Gahr M, Wieland M, Eber S. 1988. Phagocytosis-Associated functions in neonatal monocyte-derived macrophages. Pediatr Res 24: 213-216. doi:10.1203/00006450-19880800000015

Stephan AH, Barres BA, Stevens B. 2012. The complement system: an unexpected role in synaptic pruning during development and disease. Annu Rev Neurosci 35: 369-389. doi:10 .1146/annurev-neuro-061010-113810

Stevens B, Allen NJ, Vazquez LE, Howell GR, Christopherson KS, Nouri N, Micheva KD, Mehalow AK, Huberman AD, Stafford B, et al. 2007. The classical complement cascade mediates CNS synapse elimination. Cell 131: 1164-1178. doi:10.1016/ j.cell.2007.10.036

Tanabe S, Yamashita T. 2018. B-1a lymphocytes promote oligodendrogenesis during brain development. Nat Neurosci 21: 506-516. doi:10.1038/s41593-018-0106-4

Utz SG, See P, Mildenberger W, Thion MS, Silvin A, Lutz M, Ingelfinger F, Rayan NA, Lelios I, Buttgereit A, et al. 2020. Early fate defines microglia and non-parenchymal brain macrophage development. Cell 181: 557-573.e18. doi:10.1016/j .cell.2020.03.021

Van Hove H, Martens L, Scheyltjens I, De Vlaminck K, Pombo Antunes AR, De Prijck S, Vandamme N, De Schepper S, Van Isterdael G, Scott CL, et al. 2019. A single-cell atlas of mouse 
brain macrophages reveals unique transcriptional identities shaped by ontogeny and tissue environment. Nat Neurosci 22: 1021-1035. doi:10.1038/s41593-019-0393-4

Volpe JJ. 2019. Dysmaturation of premature brain: importance, cellular mechanisms, and potential interventions. Pediatr Neurol 95: 42-66. doi:10.1016/j.pediatrneurol.2019.02.016

Winterberg T, Vieten G, Meier T, Yu Y, Busse M, Hennig C, Hansen G, Jacobs R, Ure BM, Kuebler JF. 2015. Distinct phenotypic features of neonatal murine macrophages. Eur I Immunol 45: 214-224. doi:10.1002/eji.201444468

Yamada M, Kubo H, Kobayashi S, Ishizawa K, He M, Suzuki T, Fujino N, Kunishima H, Hatta M, Nishimaki K, et al. 2011. The increase in surface CXCR4 expression on lung extravascular neutrophils and its effects on neutrophils during endotoxin-induced lung injury. Cell Mol Immunol 8: 305-314. doi:10.1038/cmi.2011.8

Yilmaz G, Arumugam TV, Stokes KY, Granger DN. 2006. Role of $\mathrm{T}$ lymphocytes and interferon- $\gamma$ in ischemic stroke. Circulation 113: 2105-2112. doi:10.1161/CIRCULATIONAHA.105 .593046
Zelco A, Rocha-Ferreira E, Nazmi A, Ardalan M, Chumak T, Nilsson G, Hagberg H, Mallard C, Wang X. 2020. Type 2 innate lymphoid cells accumulate in the brain after hypoxia-ischemia but do not contribute to the development of preterm brain injury. Front Cell Neurosci 14: 249. doi:10.3389/fncel .2020 .00249

Zhang X, Rocha-Ferreira E, Li T, Vontell R, Jabin D, Hua S, Zhou K, Nazmi A, Albertsson AM, Sobotka K, et al. 2017. $\gamma \delta$ T cells but not $\alpha \beta T$ cells contribute to sepsis-induced white matter injury and motor abnormalities in mice. I Neuroinflammation 14: 255. doi:10.1186/s12974-017-1029-9

Zhao J, Qu Y, Wu J, Cao M, Ferriero DM, Zhang L, Mu D. 2013. PTEN inhibition prevents rat cortical neuron injury after hypoxia-ischemia. Neuroscience 238: 242-251. doi:10.1016/j .neuroscience.2013.02.046

Zusso M, Methot L, Lo R, Greenhalgh AD, David S, Stifani S. 2012. Regulation of postnatal forebrain amoeboid microglial cell proliferation and development by the transcription factor Runx1. J Neurosci 32: 11285-11298. doi:10.1523/JNEUROSCI $.6182-11.2012$ 


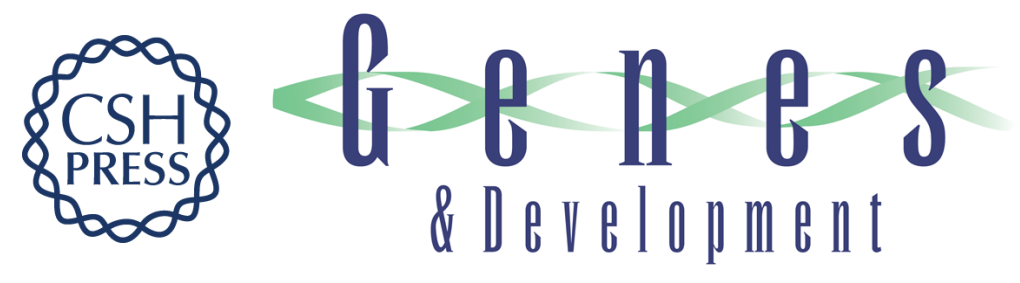

\section{Single-cell atlas reveals meningeal leukocyte heterogeneity in the developing mouse brain}

Aura Zelco, Vanja Börjesson, Jurrian K. de Kanter, et al.

Genes Dev. 2021, 35: originally published online July 22, 2021

Access the most recent version at doi:10.1101/gad.348190.120

\section{Supplemental http://genesdev.cshlp.org/content/suppl/2021/07/21/gad.348190.120.DC1 Material}

References This article cites 57 articles, 11 of which can be accessed free at: http://genesdev.cshlp.org/content/35/15-16/1190.full.html\#ref-list-1

Creative This article, published in Genes \& Development, is available under a Creative Commons Commons License (Attribution-NonCommercial 4.0 International), as described at License http://creativecommons.org/licenses/by-nc/4.0/.

Email Alerting Receive free email alerts when new articles cite this article - sign up in the box at the top Service right corner of the article or click here.

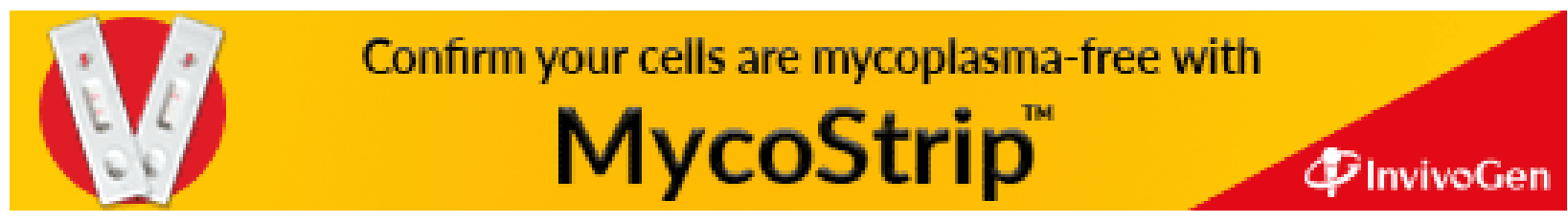

